\title{
A GENERALISATION OF THE MORSE INEQUALITIES
}

\author{
MOHAN BHUPAL
}

(Received 1 April 1999; revised 1 November 2000)

Communicated by W. Neumann

\begin{abstract}
In this paper we construct a family of variational families for a Legendrian embedding, into the 1-jet bundle of a closed manifold, that can be obtained from the zero section through Legendrian embeddings, by discretising the action functional. We compute the second variation of a generating function obtained as above at a nondegenerate critical point and prove a formula relating the signature of the second variation to the Maslov index as the mesh goes to zero. We use this to prove a generalisation of the Morse inequalities thus refining a theorem of Chekanov.
\end{abstract}

2000 Mathematics subject classification: primary 53C15; secondary 53D35.

\section{Introduction}

In [1] Arnold calls a Legendrian embedding $f: L \rightarrow J^{1} M$, into the 1-jet bundle, that can be obtained from the zero section by a smooth deformation through Legendrian embeddings a quasi-function. Intersections of $f$ with $M_{0} \times \mathbb{R}$, where $M_{0}$ denotes the zero section of $T^{*} M$, are called critical points of $f$ and these are said to be nondegenerate if the intersection is transverse. It can be shown that every such quasi-function is of the form $f=\left.\psi\right|_{M_{0} \times\{0\}}$, where $\psi=\psi^{1}$ for some contact isotopy $\left(\psi^{t}\right)_{0 \leq 1 \leq 1}$ of $J^{1} M$. Under the assumption that $M$ is closed (that is, compact and boundaryless) and a Riemannian metric on $M$ has been chosen, we show that to each nondegenerate critical point $c \in L$ of $f$ we may naturally assign a number

$$
\mu\left(c, \psi^{t}\right) \in n / 2+\mathbb{Z} \text {. }
$$

Assuming that all critical points are nondegenerate we denote by $p_{k}$ the number of critical points $c \in L$ such that $\mu\left(c, \psi^{t}\right)=k$ and by $b_{k}$ the $k$ th Betti number of $M$ (with coefficients in any field). We prove the following result.

(c) 2001 Australian Mathematical Society 0263-6115/2001 \$A2.00+0.00 
THEOREM 1.1. Let $f: L \rightarrow J^{1} M$ be a quasi-function on a closed Riemannian manifold $M$ of dimension $n$ having only nondegenerate critical points. Then

$$
p_{n / 2-k}-p_{n / 2-k+1}+\cdots \geq b_{k}-b_{k-1}+\cdots+(-1)^{k} b_{0}
$$

for every $k \in \mathbb{Z}$.

Theorem 1.1 is a refinement of a theorem by Chekanov announced by Arnold in [1] (see also Chekanov [5]). The latter theorem asserts that, in the nondegenerate case, the number of critical points is bounded from below by the sum of the Betti numbers. A proof of Chekanov's Theorem is also given by Chaperon [4] and is based on a result by Théret [16] concerning the existence of a generating function. Our proof is independent of their work.

A method of generating contact isotopies is based on using a time-dependent Hamiltonian $H:[0,1] \times J^{1} M \rightarrow \mathbb{R}$. Indeed all contact isotopies may be obtained in this way. A special instance is given by the following: given a Morse function $f: M \rightarrow \mathbb{R}$, let $H(x, y, z)=-f(x)$ define a time-independent Hamiltonian. The critical points of $f$ are in one-to-one correspondence with the critical points of the associated quasi-function. In particular, the Morse index $\operatorname{ind}_{H}\left(x_{0}\right)$ of a critical point $x_{0} \in M$ of $f$ is related to the Maslov index by

$$
\mu\left(x_{0}, \psi_{H}^{t}\right)=n / 2-\operatorname{ind}_{H}\left(x_{0}\right)
$$

and thus in this special case Theorem 1.1 reduces to the classical Morse inequalities.

Our methods also refine a related theorem in the symplectic setting proved by Hofer [9] and Laudenbach and Sikorav [11]. This pertains to finding a lower bound for the number of points of intersection of the zero section of the cotangent bundle with its image under a time-dependent Hamiltonian symplectomorphism. An outline of our approach follows.

Let $\psi^{\prime}$ be a contact isotopy of $J^{1} M$ with Hamiltonian $H:[0,1] \times J^{1} M \rightarrow \mathbb{R}$. When $M=\mathbb{R}^{n}$ we may associate with $H$ an action functional as follows. Consider the path space

$$
\mathscr{P}=\left\{c=(x, y):[0,1] \rightarrow T^{*} \mathbb{R}^{n} \mid y(0)=0\right\}
$$

and, given $c \in \mathscr{P}$, let $z=z_{c}:[0,1] \rightarrow \mathbb{R}$ be the unique solution of

$$
\dot{z}=\langle y, \dot{x}\rangle-H(t, x, y, z), \quad z(0)=0
$$

(assuming such a solution exists). Define the action functional $\Phi_{H}: \mathscr{P} \rightarrow \mathbb{R}$ by

$$
\Phi_{H}(c)=\int_{0}^{1}(\langle y, \dot{x}\rangle-H(t, x, y, z)) d t .
$$


A calculation shows that the fibre critical points of $\Phi_{H}$ with respect to the fibration $\mathscr{P} \rightarrow \mathbb{R}^{n}:(x, y) \mapsto x(1)$ generate the Legendrian submanifold $\psi^{1}\left(L_{0}\right)$.

We consider discretisations of the form $\Phi_{H}^{N}: \mathscr{P}^{N} \rightarrow \mathbb{R}$ for $N \in \mathbb{N}$ which also have this property. Here

$$
\mathscr{P}^{N}=\left\{c^{N}=\left(x_{0}, \ldots, x_{N}, y_{1}, \ldots, y_{N}\right) \in \mathbb{R}^{(2 N+1) n} \mid x_{i}, y_{j} \in \mathbb{R}^{n}\right\},
$$

which is fibred over $\mathbb{R}^{n}$ by $\mathscr{P}^{N} \rightarrow \mathbb{R}^{n}: c^{N} \mapsto x_{N}$, can be thought of as a space of discrete paths in $\mathbb{R}^{2 k}$, and $\Phi_{H}^{N}$ is the discrete action functional which is given by

$$
\Phi_{H}^{N}\left(c^{N}\right)=\sum_{j=1}^{N}\left(\left\langle y_{j}, x_{j}-x_{j-1}\right\rangle-V_{j-1}\left(x_{j-1}, y_{j}, z_{j-1}\right)\right) .
$$

The functions $V_{j}: \mathbb{R}^{2 n+1} \rightarrow \mathbb{R}$ are constructed by using the contact isotopy $\psi^{r}$, and the $z_{j} \mathrm{~S}$ are given by the iterative rule

$$
z_{0}=0, \quad z_{j}=\left\langle y_{j}, x_{j}-x_{j-1}\right\rangle-V_{j-1}\left(x_{j-1}, y_{j}, z_{j-1}\right)+z_{j-1}, \quad j>0 .
$$

We show that such discretisations always exist for $N$ sufficiently large and $H$ having compact support.

A path $c \in \mathscr{P}$ is a critical point of $\Phi_{H}$ if and only if $\left(c, z_{c}\right)$ is a trajectory of $\psi^{t}$ starting in $L_{0} \times\{0\}$, where $L_{0}$ denotes the zero section of $T^{*} \mathbb{R}^{n}$, and ending in $L_{0} \times \mathbb{R}$. Similarly, a discrete path $c^{N} \in \mathscr{P}^{N}$ is a critical point of $\Phi^{N}$ if and only if $\left(c^{N},\left\{z_{j}\right\}\right)$ corresponds to a 'broken trajectory' of $\psi^{t}$ with $\left(x_{0}, y_{0}, z_{0}\right) \in L_{0} \times\{0\}$ and $\left(x_{N}, y_{N}, z_{N}\right) \in L_{0} \times \mathbb{R}$. Furthermore, given such a path $c^{N}$, critical for $\Phi^{N}$, and such that $\psi^{1}\left(L_{0} \times\{0\}\right)$ intersects $L_{0} \times \mathbb{R}$ transversally at $\left(x_{N}, y_{N}, z_{N}\right), d^{2} \Phi_{H}^{N}\left(c^{N}\right)$ will be nondegenerate. We show that, given a critical point $c \in \mathscr{P}$ with the same property, for sufficiently large $N$ the signature of the second variation of the discretisation is given by:

Proposition 1.2. $\quad \operatorname{sign} d^{2} \Phi_{H}^{N}\left(c^{N}\right)=2 \mu\left(c^{N}, \psi^{t}\right)$.

This generalises a theorem of Robbin and Salamon in [14] for quadratic Hamiltonians. Also, it strengthens the theorem of Viterbo [17] which states that, in the symplectic case, the difference of the signature of the second variation of $\Phi^{N}$ at two critical points is independent of $N$, but at the cost of having to take $N$ sufficiently large.

The action functional and its discretisation are extended to the 1-jet bundle of a closed manifold $M$ as follows. First, $M$ is embedded in $\mathbb{R}^{k}$ for a suitable $k$. Next, a lift $\tilde{H}: J^{1} \mathbb{R}^{k} \rightarrow \mathbb{R}$ of $H$ is chosen such that $\left.\widetilde{H}\right|_{J^{\prime} \mathbb{R}^{k} \mid M}=H \circ \rho$, where $\rho:\left.J^{1} \mathbb{R}^{k}\right|_{M} \rightarrow J^{1} M$ is the natural projection map. Finally, the appropriate action functional is defined to be $\Phi_{\tilde{H}}$ restricted to the space of paths having $x(1) \in M$, and similarly for the discretisation. Theorem 1.1 follows from generalising Proposition 1.2 and appealing to the stabilised Morse theory (the Conley index). 


\section{Contact structures}

In this section we recall some basic notions of contact structures. A contact structure on a $2 n+1$-dimensional manifold $Q$ is a maximally nonintegrable field of hyperplanes $\xi \subset T Q$. If we assume that $\xi$ is transversally orientable, that is, $\xi$ is given by the kernel of some 1 -form $\alpha$, then the contact condition can be stated as

$$
\alpha \wedge(d \alpha)^{n} \neq 0 .
$$

In this case $\alpha$ is said to be a contact form for the contact structure $\xi$. An important equivalent formulation of the contact condition (1) is that $d \alpha$ is a nondegenerate 2-form on $\xi$.

Given a contact manifold $(Q, \xi)$ and an integral submanifold $L \subset Q$ note that, for each $p \in Q$, the tangent space $T_{p} L$ is an isotropic subspace of the symplectic vector space $\left(\xi_{p}, d \alpha_{p}\right)$, that is, $d \alpha_{p}$ vanishes on $T_{p} L$. This implies, in particular, that the dimension of $L$ can be at most $n$. In such a case $L$ is said to be Legendrian.

A diffeomorphism $\psi: Q \rightarrow Q$ which preserves the oriented field of hyperplanes $\xi$ is called a contactomorphism, that is, equivalently, if

$$
\psi^{*} \alpha=e^{g} \alpha
$$

for some function $g: Q \rightarrow \mathbb{R}$.

Note that if $\psi: Q \rightarrow Q$ is a contactomorphism, then, by a direct calculation, its derivative restricts to give a linear conformal symplectomorphism

$$
\left.d \psi(p)\right|_{\xi_{p}}:\left(\xi_{p}, d \alpha_{p}\right) \rightarrow\left(\xi_{\psi(p)}, d \alpha_{\psi(p)}\right),
$$

for every $p \in Q$.

A smooth family $\left(\psi^{t}\right)_{0 \leq r \leq 1}$ of diffeomorphisms $\psi^{t}: Q \rightarrow Q$ with $\psi^{0}=$ id is called an isotopy of $Q$. When each $\psi^{\prime}$ is a contactomorphism, the family $\psi^{\prime}$ is called a contact isotopy.

A contact vector field is defined to be a vector field $X: Q \rightarrow T Q$ which satisfies

$$
\mathscr{L}_{X} \alpha=h \alpha
$$

for some function $h: Q \rightarrow \mathbb{R}$.

The contact Hamiltonian associated to a contact vector field $X: Q \rightarrow T Q$ is defined to be the function $H: M \rightarrow \mathbb{R}$ given by

$$
H=-\iota(X) \alpha .
$$

It is easy to check that every function $H: Q \rightarrow \mathbb{R}$ is the contact Hamiltonian of a unique contact vector field $X=X_{H}: Q \rightarrow T Q$. 
1-jet bundles. The standard contact structure on the 1 -jet bundle $J^{1} M=T^{*} M \times \mathbb{R}$ of an $n$-dimensional manifold $M$ is given as kernel of the 1 -form

$$
\alpha=d z-\lambda_{\text {can }} \text {. }
$$

Here $z$ denotes the standard coordinate on $\mathbb{R}$ and $\lambda_{\text {can }}$ the canonical 1-form on $T^{*} M$ which is given by the defining property $\sigma^{*} \lambda_{\text {can }}=\sigma$ for every 1 -form $\sigma: M \rightarrow T^{*} M$.

Let $\psi^{t}$ be a contact isotopy of $J^{1} M$ and $X:[0,1] \times J^{1} M \rightarrow T J^{1} M$ be the associated time-dependent contact vector field defined by

$$
\frac{d}{d t} \psi^{t}(p)=X\left(t, \psi^{t}(p)\right)
$$

Associated to $X$ is the time-dependent Hamiltonian $H:[0,1] \times J^{1} M \rightarrow \mathbb{R}$ given by (2). We express this in local coordinates.

About any point $(c, z) \in J^{1} M$ one can construct a distinguished set of local coordinates $x_{1}, \ldots, x_{n}, y_{1}, \ldots, y_{n}, z$ in which $\alpha$ takes the form

$$
\alpha=d z-\sum_{i} y_{i} d x_{i} \text {. }
$$

Using these coordinates it is easy to check that $X$ is given by Hamilton's equations:

$$
\begin{aligned}
\dot{x}_{i} & =\frac{\partial H}{\partial y_{i}} \\
\dot{y}_{i} & =-\frac{\partial H}{\partial x_{i}}-y_{i} \frac{\partial H}{\partial z} \\
\dot{z} & =\langle y, \dot{x}\rangle-H
\end{aligned}
$$

and thus $\psi^{t}$ is the time- $t$ map of the flow associated to Hamilton's equations for $H$.

\section{The variational principle}

In this section we describe an abstract method for generating all solutions of Hamilton's equations in the 1 -jet bundle $J^{1} M$ subject to $c(0) \in M_{0}$, the zero section of $T^{*} M$, and $z(0)=0$. Let $H:[0,1] \times J^{1} M \rightarrow \mathbb{R}$ be a time-dependent Hamiltonian. To ensure that (4) below has a solution $z:[0,1] \rightarrow \mathbb{R}$ for every smooth path $c:[0,1] \rightarrow$ $T^{*} M$ assume that $\partial_{z} H$ is bounded. Let $\pi: \mathscr{P}_{M} \rightarrow M$ be the fibre bundle, where

$$
\mathscr{P}_{M}=\left\{c:[0,1] \rightarrow T^{*} M \mid c(0) \in M_{0}\right\}
$$

is a space of smooth paths and $\pi$ is the projection $c \mapsto \pi_{T^{*} M} c(1) \in M$. To each path $c \in \mathscr{P}_{M}$ associate a function $z=z_{c}:[0,1] \rightarrow \mathbb{R}$ given as the unique solution of

$$
\dot{z}(t)=\lambda_{\text {can }}(\dot{c}(t))-H(t, c(t), z(t)), \quad z(0)=0 .
$$


Now define the action functional $\Phi_{H}: \mathscr{P}_{M} \rightarrow \mathbb{R}$ by

$$
\Phi_{H}(c)=z(1)=\int_{0}^{1}\left(\lambda_{\text {can }}(\dot{c})-H(t, c, z)\right) d t .
$$

A point $c \in \mathscr{P}_{M}$ is called a fiber critical point of $\Phi_{H}$ if the differential of $\Phi_{H}$ disappears along $T_{c}\left(\mathscr{P}_{M}\right)_{\pi(c)}$, the tangent space at $c$ of the fibre over $\pi(c)$.

PROPOSITION 3.1. There is a one-to-one correspondence between the set of fibre critical points of $\Phi_{H}$ and the solutions of Hamilton's equations subject to $c(0) \in M_{0}$, $z(0)=0$. Furthermore, $c \in \mathscr{P}_{M}$ is fibre critical if and only if

$$
\iota(\dot{c}) d \lambda_{\text {can }}+d H_{t, z}(c)+\partial_{z} H(t, c, z) \lambda_{\text {can }}=0 .
$$

PROOF. Let $(-\varepsilon, \varepsilon) \rightarrow \mathscr{P}_{M}: s \mapsto c_{s}$ be a parametrised family of paths and abbreviate $c_{0}=c$. Denote by $\gamma:[0,1] \rightarrow c^{*} T\left(T^{*} M\right)$ the associated variational field

$$
\gamma(t)=\left.\frac{\partial}{\partial s}\right|_{0} c_{s}(t)
$$

Define $z_{:}:[0,1] \rightarrow \mathbb{R}$ for each $s$ by (4) and denote the derivative with respect to $s$ at $s=0$ by $\zeta$. Now $\zeta$ evaluated at $t \in[0,1]$ is given by the expression

$$
\begin{aligned}
\zeta(t) & =\left.\frac{\partial}{\partial s}\right|_{0} \int_{0}^{t} c_{s}^{*}\left(\lambda_{\mathrm{can}}-H_{z_{s}} d \tau\right) \\
& =\int_{0}^{t} c^{*} \mathscr{L}_{\gamma}\left(\lambda_{\mathrm{can}}-H_{z} d \tau\right)-\partial_{z} H \zeta d \tau \\
& =\int_{0}^{t}\left(\iota(\gamma)\left(-\iota(\dot{c}) d \lambda_{\mathrm{can}}-d H_{\tau, z}\right)-\partial_{z} H \zeta\right) d \tau+\lambda_{\mathrm{can}}(\gamma(t)),
\end{aligned}
$$

where the third equality follows from Cartan's identity for the Lie derivative. Thus the 1 -form $\alpha$ applied to the vector field $(\gamma, \zeta)$ satisfies

$$
\begin{aligned}
\alpha(\gamma(t), \zeta(t)) & =\int_{0}^{t}\left(\iota(\gamma)\left(-\iota(\dot{c}) d \lambda_{\mathrm{can}}-d H_{\tau, z}-\partial_{z} H \lambda_{\mathrm{can}}\right)-\partial_{z} H \alpha(\gamma, \zeta)\right) d \tau, \\
\alpha(\gamma(0), \zeta(0)) & =0 .
\end{aligned}
$$

This we can solve explicitly. Evaluating at $t=1$ we find

$$
\alpha(\gamma(1), \zeta(1))=\int_{0}^{1} e^{f_{t}^{1}-\partial_{z} H d \tau} \iota(\gamma)\left(-\iota(\dot{c}) d \lambda_{\text {can }}-d H_{t, z}-\partial_{z} H \lambda_{\text {can }}\right) d t .
$$

Thus it follows that $c \in \mathscr{P}_{M}$ is fibre critical if and only if (5) holds and since (5) in local coordinates is equivalent to (3a), (3b) this proves the proposition. 
Note that at a fibre critical point $c$ the differential of $\Phi_{H}$ is given by

$$
d \Phi_{H}(c)(\gamma)=\lambda_{\text {can }}(\gamma(1)) .
$$

It thus follows that $c$ is a critical point only if $c(1) \in M_{0}$.

\section{Variational families}

The notion of a variational family formalises the variational principle that was considered in the previous section. In this section we consider some basic properties of variational families in general. We start by briefly recalling some ideas from the theory of symplectic reduction.

Suppose that $(P, \omega)$ is a symplectic manifold and that $W \subset P$ is a coisotropic submanifold. This means that $T_{p} W^{\omega}$ is contained in $T_{p} W$ for each $p \in W$, where $T_{p} W^{\omega}$ denotes the symplectic complement of $T_{p} W$. Then the subspaces $T_{p} W^{\omega}$ determine an isotropic distribution $T W^{\omega}$ in $T P$ which by the closedness of $\omega$ is integrable. It follows from Frobenius' theorem that $W$ is foliated by isotropic leaves. If we assume that the quotient space $P^{\prime}=W / \sim$ is a manifold, where the equivalence relation is given by: $p_{0} \sim p_{1}$ if $p_{0}$ and $p_{1}$ lie in the same leaf, then we note that the $P^{\prime}$ is naturally a symplectic manifold.

We next recall that if $L$ is a Lagrangian submanifold of $P$ which intersects $W$ cleanly, that is, $L \cap W$ is a submanifold of $P$ and $T_{p}(L \cap W)=T_{p} L \cap T_{p} W$ for all $p \in L \cap W$, then the image of $L$ in $P^{\prime}$ is also Lagrangian, but may only be immersed.

To pass from Lagrangian immersions to Legendrian immersions we use the process of contactisation. This applies to all symplectic manifolds $(P, \omega)$, where the symplectic form is given as the differential of a 1 -form $-\lambda$. Such manifolds are said to be exact. A contactisation of an exact symplectic manifold $(P, \omega)$ is a contact manifold $(P \times \mathbb{R}, d z-\lambda)$, where $\lambda$ is a 1 -form satisfying the relation $\omega=-d \lambda$. Here $z$ denotes the coordinate on $\mathbb{R}$. Given an exact symplectic manifold $(P, \omega)$, a contactisation of a Lagrangian immersion $f: L \rightarrow P$ is a Legendrian immersion $\tilde{f}: L \rightarrow P \times \mathbb{R}$ lifting $f$. If the Lagrangian immersion $f: L \rightarrow P$ is exact, that is, $f^{*} \lambda=d S$ for some function $S: L \rightarrow \mathbb{R}$, then the lift $L \rightarrow P \times \mathbb{R}: c \mapsto(f(c), S(c))$ is Legendrian. Conversely, it is easy to see that if $f$ admits a lift, then it must be exact. Note, however, that the contactisation of an exact Lagrangian immersion is not unique since the function $S$ is unique only up to the addition of a locally constant function.

We now give the definition of a variational family. This is a pair consisting of a fibre bundle $\pi: E \rightarrow X$ and a smooth function $\Phi: E \rightarrow \mathbb{R}$. The variational family is called transversal if the graph of $d \Phi$ in $T^{*} E$ intersects the conormal bundle of the fibres

$$
N_{E}=\left\{(c, \eta) \in T^{*} E \mid \eta \in(\operatorname{ker} d \pi(c))^{\perp}\right\}
$$


transversally. For a transversal variational family $(E, \Phi)$, the set of fibre critical points is given by

$$
\mathscr{C}_{E, \Phi}=\left\{c \in E \mid d \Phi(c) \in(\operatorname{ker} d \pi(c))^{\perp}\right\}
$$

which by transversality is a manifold. To each fibre critical point we can uniquely associate a covector $v^{*} \in T_{\pi(c)}^{*} X$ by the relation $v^{*} \circ d \pi(c)=d \Phi(c)$. This gives rise to a map $\iota_{\Phi}: \mathscr{C}_{E, \Phi} \rightarrow J^{1} X$ given by mapping $c \in \mathscr{C}_{E, \Phi}$ to the 1 -jet $\left(\pi(c), v^{*}, \Phi(c)\right)$.

The following proposition is due to Hörmander [10]. We include here a proof for the sake of completeness.

PROPOSITION 4.1. Given a transversal variational family $(E, \Phi)$, the map $\iota_{\Phi}: \mathscr{C}_{E, \Phi}$ $\rightarrow J^{\prime} M$ is a Legendrian immersion.

PROOF. Note that the graph of $d \Phi$ is a Lagrangian submanifold of $T^{*} E$ and $N_{E}$ is a coisotropic submanifold. Note also that the quotient $N_{E} / \sim$ can be identified with $T^{*} X$. It follows from the above discussion that the map $\iota_{\Phi}^{\prime}: \mathscr{C}_{E, \Phi} \rightarrow T^{*} X$, given by composing $\iota_{\Phi}$ with the projection $J^{1} X \rightarrow T^{*} X$, is a Lagrangian immersion. Moreover, the pullback of $\lambda_{\text {can }}$ by $\iota_{\Phi}^{\prime}$ is given by

$$
\iota_{\Phi}^{*} \lambda_{\text {can }}=v^{*} \circ d\left(\left.\pi\right|_{\mathscr{C}_{E, \Phi}}\right)=d\left(\left.\Phi\right|_{\mathscr{C}_{E, \Phi}}\right)
$$

and so $\iota_{\Phi}^{\prime}$ is an exact immersion. Contactising, it follows that $\iota_{\Phi}$ is a Legendrian immersion.

Denote by $L_{\Phi}$ the image of $\iota_{\Phi}$ and by $X_{0}$ the zero section of $T^{*} X$. Clearly, a point $c \in \mathscr{C}_{E . \Phi}$ is a critical point of $\Phi$ if and only if $\iota_{\Phi}(c)$ is in $L_{\Phi} \cap X_{0} \times \mathbb{R}$. Moreover, when $E$ is a finite dimensional vector bundle we have:

LEMMA 4.2. A point $c \in$ crit $\Phi$ is a nondegenerate critical point of $\Phi$ if and only if $L_{\Phi}$ and $X_{0} \times \mathbb{R}$ intersect transversally at $\iota_{\Phi}(c)$.

The proof of this uses the following elementary fact.

LEMMA 4.3. Let $(V, \omega)$ be a symplectic vector space and $N \subset V$ a coisotropic subspace. Then given two Lagrangian subspaces $\Lambda_{0}$ and $\Lambda_{1}$ satisfying

$$
\Lambda_{0} \subset N, \quad \Lambda_{1} \cap N^{\omega}=\{0\}
$$

$\Lambda_{0}$ is transverse to $\Lambda_{1}$ if and only if, in the quotient, the reduced spaces $\bar{\Lambda}_{0}$ and $\bar{\Lambda}_{1}$ are transversal. 
PROOF OF LemMA 4.2. Apply Lemma 4.3 in case $V=T_{p}\left(T^{*} E\right), N=T_{p} N_{E}$, $\Lambda_{0}=T_{p} E_{0}$ and $\Lambda_{1}=T_{p}(\operatorname{graph}(d \Phi))$ where $p=(c, d \Phi(c))$ and $E_{0}$ denotes the zero section of $T^{*} E$.

EXAMPLE 4.4 (Action). Let $H:[0,1] \times J^{1} M \rightarrow \mathbb{R}$ be a time-dependent Hamiltonian and $\Phi_{H}: \mathscr{P}_{M} \rightarrow \mathbb{R}$ the action functional given in Section 3. By Proposition 3.1 the pair $\left(\mathscr{P}_{M}, \Phi_{H}\right)$ is a variational family for the Legendrian submanifold $\psi^{1}\left(M_{0} \times\{0\}\right)$, where $\psi^{t}=\psi_{H}^{t}$ denotes the contact isotopy generated by $H$. Note that we do not say anything about transversality.

Restricted variational families. Let $M$ be a submanifold of $X$ and let $L \rightarrow$ $J^{1} X: c \mapsto(f(c), S(c))$ be a Legendrian immersion transversal to $\left.J^{1} X\right|_{M}$. We extend the notion of symplectic reduction to such Legendrian immersions as follows.

Evidently $f: L \rightarrow T^{*} X$ is an exact Lagrangian immersion. Define $f^{\prime}: L^{\prime} \rightarrow$ $T^{*} M$, where $L^{\prime}=f^{-1}\left(\left.T^{*} X\right|_{M}\right)$, by $f^{\prime}=r \circ f \circ i$. Here $i: L^{\prime} \hookrightarrow L$ is the inclusion and $r:\left.T^{*} X\right|_{M} \rightarrow T^{*} M$ is the natural projection. By transversality $L^{\prime}$ is a manifold of the same dimension as $M$. Using $r^{*} \lambda_{\text {can }}=j^{*} \lambda_{\text {can }}$, where $j:\left.T^{*} X\right|_{M} \hookrightarrow T^{*} X$ is the inclusion, it is now easy to see that $f^{\prime}$ is again an exact Lagrangian immersion with $f^{\prime *} \lambda_{\text {can }}=d(S \circ i)$. The lift $L^{\prime} \rightarrow J^{1} M: c \mapsto\left(f^{\prime}(c), S \circ i(c)\right)$ we refer to as the reduction of $(f, S)$.

Suppose that $(\pi: E \rightarrow X, \Phi: E \rightarrow \mathbb{R})$ is a transversal variational family and denote by

$$
\left(\pi_{M}=\left.\pi\right|_{E_{M}}: E_{M} \rightarrow M, \Phi_{M}=\left.\Phi\right|_{E_{M}}: E_{M} \rightarrow \mathbb{R}\right),
$$

where $E_{M}=\left.E\right|_{M}$, the variational family obtained by restricting to $M$. In the case of a finite dimensional vector bundle these are related by:

LEMMA 4.5. Given a transversal variational family $(E, \Phi)$, the restricted variational family $\left(E_{M}, \Phi_{M}\right)$ is transversal if and only if $L_{\Phi}$ is transverse to $\left.J^{1} X\right|_{M}$. Moreover, if both variational families are transversal, then $L_{\Phi_{M}}$ is the reduction of $L_{\Phi}$.

PROOF. The first part of the lemma follows easily by choosing local coordinates on $X$ in which $M$ is linear and the second part follows by a direct calculation.

COROLlaRY 4.6. If both $(E, \Phi)$ and $\left(E_{M}, \Phi_{M}\right)$ are transversal, then $c \in E_{M}$ is a nondegenerate critical point of $\Phi_{M}$ if and only if $L_{\Phi}$ and $T M^{\perp} \times \mathbb{R}$, where $T M^{\perp} \subset T^{*} X$ denotes the conormal bundle of $M$, intersect transversally at $\iota_{\Phi}(c)$.

PROOF. This follows immediately from Lemma 4.2, Lemma 4.3 and Lemma 4.5. 


\section{Continuous-time theory}

In this section we assume that $M$ is a closed manifold embedded in $\mathbb{R}^{k}$ and that $\psi^{\prime}: J^{1} M \rightarrow J^{1} M$ is a given contact isotopy. We construct a variational family $\left(\mathscr{P}_{\mathbb{R}^{k}}, \Phi_{H}\right)$ on $\mathbb{R}^{k}$ whose restriction to $M,(\mathscr{P}, \Phi)$, generates the Legendrian submanifold $\psi^{1}\left(M_{0} \times\{0\}\right) \subset J^{1} M$, and consider the second variation of the function $\Phi$.

5.1. Construction of variational family. Suppose that $H:[0,1] \times J^{1} \mathbb{R}^{k} \rightarrow \mathbb{R}$ is a time-dependent Hamiltonian with the property that for every $x \in M$

$$
H(t, x, y, z)=H\left(t, x, y^{\prime}, z\right) \quad \text { whenever } \quad y-y^{\prime} \perp T_{x} M .
$$

Denoting by $\rho:\left.J^{1} \mathbb{R}^{k}\right|_{M} \rightarrow J^{1} M$ the natural projection $(x, y, z) \mapsto\left(x,\left.y\right|_{T_{x} M}, z\right)$, it follows that $H$ descends to a time-dependent Hamiltonian $\bar{H}:[0,1] \times J^{1} M \rightarrow \mathbb{R}$ given by

$$
\left.H\right|_{\left.J^{\prime} \mathbb{R}^{k}\right|_{M}}=\bar{H} \circ \rho .
$$

We insist that $H_{t}$ is $C^{1}$-bounded for every $t \in[0,1]$. This ensures that the Hamiltonian flows of $H$ and $\bar{H}$ define global diffeomorphisms for all $t \in[0,1]$.

The next lemma shows how the Hamiltonian flow of $H$ is related to that of $\bar{H}$ (compare with [2]).

LEMMA 5.1. Let $H$ be a time-dependent Hamiltonian on $J^{1} \mathbb{R}^{k}$ and denote by $\varphi^{t}$ the associated contact isotopy. Then the following are equivalent:

(i) $H$ satisfies (6) for some function $\bar{H}:[0,1] \times J^{1} M \rightarrow \mathbb{R}$;

(ii) $\varphi^{t}$ leaves $\left.J^{1} \mathbb{R}^{k}\right|_{M}$ invariant;

(iii) $\varphi^{t}$ descends to a contact isotopy on $J^{1} M$.

Proof. (i) implies (ii). Let $X=(\dot{x}, \dot{y}, \dot{z})$ denote the vector field generated by $H$. It is sufficient to show that $X_{t}(p) \in T_{p}\left(\left.J^{1} \mathbb{R}^{k}\right|_{M}\right)$ for all $\left.p \in J^{1} \mathbb{R}^{k}\right|_{M}$. To see this note that, for any $p=\left.(x, y, z) \in J^{1} \mathbb{R}^{k}\right|_{M}$,

$$
\dot{x}_{t}(p)=\partial_{y} H_{t}(x, y, z)=\partial_{y} H_{t}(x, \Pi(x) y, z)=\Pi(x) \partial_{y} H_{t}(x, y, z) .
$$

Here $T^{*} M$ has been identified with $T M$, using the induced inner product, and $\Pi(x) \in$ $\mathbb{R}^{k \times k}$ denotes the orthogonal projection onto $T_{x} M$. It follows that $\dot{x}_{i}(p) \in T_{x} M$ and hence $X_{t}(p) \in T_{p}\left(\left.J^{1} \mathbb{R}^{k}\right|_{M}\right)$ as required.

(ii) implies (iii). We first claim that if a contactomorphism $\varphi$ preserves $\left.J^{1} \mathbb{R}^{k}\right|_{M}$, then it has the property that

$$
\rho(\varphi(p))=\rho(\varphi(q)) \text { whenever } \rho(p)=\rho(q) .
$$


To see this note that the subspace $N_{p}=T_{p}\left(\left.J^{1} \mathbb{R}^{k}\right|_{M}\right) \cap \xi_{p}$ is a coisotropic subspace of the symplectic vector space $\left(\xi_{p}, d \alpha_{p}\right)$, for each $\left.p \in J^{1} \mathbb{R}^{k}\right|_{M}$, and that the symplectic complement is given simply by $N_{p}^{\omega}=T_{p}\left(\rho^{-1}(\rho(p))\right)$. Also note that $d \varphi(p)$ restricts to a map from $N_{p}$ to $N_{\varphi(p)}$. Property (7) now follows from the fact that the fibers of $\rho$ are connected.

Consequently, we may define $\psi^{t}: J^{1} M \rightarrow J^{1} M$ by $\rho \circ \varphi^{t} \circ j=\psi^{t} \circ \rho$, where $j$ denotes the inclusion $\left.J^{1} \mathbb{R}^{k}\right|_{M} \hookrightarrow J^{1} \mathbb{R}^{k}$. Clearly $\psi^{t}$ is a diffeomorphism. That $\psi^{\prime}$ is a contactomorphism follows from a direct calculation using the identity $j^{*} \alpha=\rho^{*} \alpha$.

(iii) implies (i). Since $\varphi^{t}$ descends to a contact isotopy $\psi^{t}$ on $J^{t} M$ it is clear that $\varphi^{t}$ preserves $\left.J^{1} \mathbb{R}^{k}\right|_{M}$. Also it follows that the contact vector fields $X, \bar{X}$ generated by the isotopies $\varphi^{t}, \psi^{t}$ respectively are related by $\rho_{*} X=\bar{X}$. These satisfy $\iota(X) \alpha=$ $-H, \iota(\bar{X}) \alpha=-\bar{H}$, where $\bar{H}$ is the Hamiltonian function generating $\psi^{t}$. A direct calculation now shows that $H$ and $\bar{H}$ are related by (6). This completes the proof of the lemma.

REMARK 5.2. It follows from Lemma 5.1 that, when $H$ satisfies (6), there is a commutative diagram

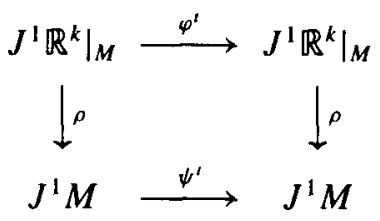

but note that $\varphi^{\prime}$ is not uniquely determined by the restriction of $H$ to $\left.J^{1} \mathbb{R}^{k}\right|_{M}$.

We now return to the main problem and assume, from now on, that our contact isotopy $\psi^{t}$ is generated by $\bar{H}$. We define the variational family $(\mathscr{P}, \Phi)$ as follows.

Let $\mathscr{P}=\mathscr{P}_{\mathbb{R}^{k}, M}$ be the space of paths

$$
\mathscr{P}=\left\{c=(x, y):[0,1] \rightarrow T^{*} \mathbb{R}^{k} \mid y(0)=0, x(1) \in M\right\}
$$

in $T^{*} \mathbb{R}^{k}$, and let $\pi$ be the projection $\mathscr{P} \rightarrow M$ given by $c=(x, y) \mapsto x(1)$. Define the action functional $\Phi=\Phi_{H, M}: \mathscr{P} \rightarrow \mathbb{R}$ by

$$
\Phi(c)=\int_{0}^{1}(\langle y(t), \dot{x}(t)\rangle-H(t, x(t), y(t), z(t))) d t,
$$

where $z:[0,1] \rightarrow \mathbb{R}$ is the unique solution of the initial value problem

$$
\dot{z}(t)=\langle y(t), \dot{x}(t)\rangle-H(t, x(t), y(t), z(t)), \quad z(0)=0 .
$$

Lemma 5.1 now implies the following. 
PROPOSITION 5.3. $(\mathscr{P}, \Phi)$ is a variational family for the Legendrian submanifold $\psi^{1}\left(M_{0} \times(0\}\right) \subset J^{\prime} M$.

In particular, $c \in \mathscr{P}$ is a critical point of $\Phi$ if and only if $(c, z)$ is a solution of Hamilton's equations and $c(1)$ is in $T M^{\perp}$, the conormal bundle of $M$.

REMARK 5.4. The variational families $\left(\mathscr{P}_{M}, \Phi_{\bar{H}}\right)$ of Example 4.4 , and $(\mathscr{P}, \Phi)$, considered above, both generate the Legendrian submanifold $\psi^{1}\left(M_{0} \times\{0\}\right) \subset J^{1} M$. These are related as follows: let $c \in \mathscr{P}$ be a fibre critical point of $\Phi$, then $\bar{c} \in \mathscr{P}_{M}$, given by composing $c$ with the projection $\left.T^{*} \mathbb{R}^{k}\right|_{M} \rightarrow T^{*} M$, is a fibre critical point of $\Phi_{\bar{H}}$. Conversely, any fibre critical point $\bar{c}$ of $\Phi_{\bar{H}}$ lifts uniquely to a fibre critical point $c$ of $\Phi$ by using the contact isotopy $\varphi^{t}$.

5.2. The second variation. From the proof of Proposition 3.1 the differential of $\Phi$ is given by

$$
d \Phi(c)(\gamma)=\int_{0}^{1} e^{\int_{t}^{1}-\partial_{z} H d z}\left(\left\langle\eta, \dot{x}-\partial_{y} H\right\rangle+\left\langle\dot{y}+\partial_{x} H+y \partial_{z} H, \xi\right\rangle\right) d t+\langle y(1), \xi(1)\rangle,
$$

where $\gamma \in T_{c} \mathscr{P}=\left\{\gamma=(\xi, \eta):[0,1] \rightarrow \mathbb{R}^{2 k} \mid \eta(0)=0, \xi(1) \in T_{x(1)} M\right\}$. In order to deal with the boundary term $\langle y(1), \xi(1)\rangle$ we now restrict $\Phi$ to the space

$$
\mathscr{E}=\mathscr{E}_{\mathbb{R}^{\lambda}, M}=\left\{c \in \mathscr{P} \mid c(1) \in T M^{\perp}\right\}
$$

of paths satisfying Lagrangian boundary conditions. Before giving a formula for the second variation of $\Phi$ at a critical point we introduce some notation.

Let $c \in \mathscr{P}$ be a critical point of $\Phi$ and denote by $z:[0,1] \rightarrow \mathbb{R}$ the corresponding solution of (8). Define, for reasons that will become apparent later, the matrix valued functions $G_{\xi \xi}, G_{\xi \eta}, G_{\eta \xi}, G_{\eta \eta}:[0,1] \rightarrow \mathbb{R}^{k \times k}$ and $G_{\zeta}:[0,1] \rightarrow \mathbb{B}$ by

$$
\begin{aligned}
G_{\xi \xi} & =\partial_{x x}^{2} H+\partial_{x z}^{2} H y^{T}+y \partial_{z x}^{2} H+\partial_{z z}^{2} H y y^{T}, \\
G_{\xi \eta} & =\partial_{x y}^{2} H+y \partial_{z y}^{2} H, \\
G_{\eta \xi} & =\partial_{y x}^{2} H+\partial_{y z}^{2} H y^{T}, \\
G_{\eta \eta} & =\partial_{y y}^{2} H, \\
G_{\zeta} & =\partial_{z} H .
\end{aligned}
$$

Here the derivatives of $H$ are evaluated at $(t, c(t), z(t)) \in[0,1] \times J^{1} \mathbb{R}^{k}$. Note that $G_{\xi \xi}(t)$ and $G_{\eta \eta}(t)$ are symmetric and that $G_{\xi \eta}(t)=G_{\eta \xi}(t)^{T}$. Construct from these the symmetric matrix valued function $S=S_{G}:[0,1] \rightarrow \mathbb{R}^{2 k \times 2 k}$ by

$$
S(t)=\left(\begin{array}{cc}
G_{\xi \xi}(t) & G_{\xi \eta}(t)+\frac{1}{2} G_{\zeta}(t) \mathbb{1} \\
G_{\eta \xi}(t)+\frac{1}{2} G_{\zeta}(t) \mathbb{1} & G_{\eta \eta}(t)
\end{array}\right) .
$$


Also, denote by $J_{0}$ the $2 k \times 2 k$ matrix

$$
J_{0}=\left(\begin{array}{rr}
0 & -\mathbb{1} \\
1 & 0
\end{array}\right)
$$

and by $\mathscr{C}_{c}=\mathscr{C}_{c, M}$ the tangent space of $\mathscr{E}$ at $c$ :

$$
\mathscr{C}_{c}=\left\{\gamma:[0,1] \rightarrow \mathbb{R}^{2 k} \mid \gamma(0) \in \Lambda_{0}, \gamma(1) \in \Lambda_{1}\right\}
$$

Here $\Lambda_{0}=\mathbb{R}^{k} \times\{0\}$ and $\Lambda_{1}=T_{c(1)}\left(T M^{\perp}\right)$ are Lagrangian subspaces of $\mathbb{R}^{2 k}$.

PROPOSITION 5.5. The second differential of $\Phi$ at a critical point $c$ is of the form

$$
d^{2} \Phi(c)\left(\gamma_{1}, \gamma_{2}\right)=\left\langle A(c) \gamma_{1}, \gamma_{2}\right\rangle_{L^{2}}
$$

where $\gamma_{1}, \gamma_{2} \in \mathscr{C}_{c}$ and the second variation, $A(c)$, is given by

$$
A(c) \gamma=e^{\int_{r}^{1}-G_{\zeta} d \tau}\left(J_{0} \dot{\gamma}-S \gamma+\frac{1}{2} G_{\zeta} J_{0} \gamma\right)
$$

PROOF. Fix a two parameter family of curves

$$
c_{s_{1}, s_{2}}=\left(x_{s_{1}, s_{2}}, y_{s_{1}, s_{2}}\right) \in \mathscr{E}, \quad s_{1}, s_{2} \in(-\varepsilon, \varepsilon)
$$

with $c_{0,0}=c$ and denote the derivatives by

$$
\xi_{i, s_{1}, s_{2}}=\frac{\partial}{\partial s_{i}} x_{s_{1}, s_{2}}, \quad \eta_{i, s_{1}, s_{2}}=\frac{\partial}{\partial s_{i}} y_{s_{1}, s_{2}}, \quad \zeta_{i, s_{1}, s_{2}}=\frac{\partial}{\partial s_{i}} z_{s_{1}, s_{2}}
$$

for $i=1,2$. Here, of course, $z_{s_{1}, s_{2}}$ is given by solving (8) with $x, y, z$ replaced by $x_{s_{1}, s_{2}}$, $y_{s_{1}, s_{2}}, z_{s_{1}, s_{2}}$. Abbreviate $\xi_{i}=\xi_{i, 0,0}, \eta_{i}=\eta_{i, 0,0}, \zeta_{i}=\zeta_{i, 0,0}$ and set $\gamma_{i}=\left(\xi_{i}, \eta_{i}\right), \mu_{i}=$ $\left\{\xi_{i}, \eta_{i}, \xi_{i}\right\}$. We now proceed with the proof of the proposition.

Differentiate the identity

$$
\dot{z}_{s_{1}, s_{2}}=\left\langle y_{s_{1}, s_{2}}, \dot{x}_{s_{1}, s_{2}}\right\rangle-H\left(\cdot, x_{s_{1}, s_{2}}, y_{s_{1}, s_{2}}, z_{s_{1}, s_{2}}\right)
$$

with respect to $s_{2}$ and $s_{1}$ successively and then evaluate at $s_{1}=s_{2}=0$ to obtain

$$
\begin{aligned}
\partial_{s_{1}} \dot{\zeta}_{2}= & \left\langle\partial_{s_{1}} \eta_{2}, \dot{x}\right\rangle+\left\langle\eta_{2}, \dot{\xi}_{1}\right\rangle+\left\langle\eta_{1}, \dot{\xi}_{2}\right\rangle+\left\langle y, \partial_{s_{1}} \dot{\xi}_{2}\right\rangle \\
& -d^{2} H_{r}\left(\mu_{1}, \mu_{2}\right)-\left\langle\partial_{x} H, \partial_{s_{1}} \xi_{2}\right\rangle-\left\langle\partial_{y} H, \partial_{s_{1}} \eta_{2}\right\rangle-\partial_{z} H \partial_{s_{1}} \zeta_{2}
\end{aligned}
$$

Using the fact that $c$ is critical rewrite this as

$$
\dot{\zeta}=\left\langle\eta_{2}, \dot{\xi}_{1}\right\rangle+\left\langle\eta_{1}, \dot{\xi}_{2}\right\rangle+\frac{d}{d t}\left\langle y, \partial_{s_{1}} \xi_{2}\right\rangle+\left\langle y \partial_{z} H, \partial_{s_{1}} \xi_{2}\right\rangle-\partial_{z} H \zeta-d^{2} H_{t}\left(\mu_{1}, \mu_{2}\right),
$$


where $\zeta=\partial_{s_{1}} \zeta_{2}$ or more concisely, by using the substitution $\lambda=\zeta-\left\langle y, \partial_{s_{1}} \xi_{2}\right\rangle$, as

$$
\frac{d}{d t} \lambda=\left\langle\eta_{2}, \dot{\xi}_{1}\right\rangle+\left\langle\eta_{1}, \dot{\xi}_{2}\right\rangle-\partial_{z} H \lambda-d^{2} H_{t}\left(\mu_{1}, \mu_{2}\right)
$$

Since $\lambda(0)=0$ the solution to this ODE evaluated at $t=1$ is

$$
\lambda(1)=\int_{0}^{1} e^{\int_{t}^{1}-G_{\zeta} d \tau}\left(\left\langle\eta_{2}, \dot{\xi}_{1}\right\rangle+\left\langle\eta_{1}, \dot{\xi}_{2}\right\rangle-d^{2} H_{t}\left(\mu_{1}, \mu_{2}\right)\right) d t .
$$

Also since $0=\partial_{s_{1}}\left\langle y(1), \xi_{2}(1)\right\rangle=\left\langle\eta_{1}(1), \xi_{2}(1)\right\rangle+\left\langle y(1), \partial_{s_{1}} \xi_{2}(1)\right\rangle$ it follows that $\lambda(1)=\zeta(1)+\left\langle\eta_{1}(1), \xi_{2}(1)\right\rangle$. Now partially integrate the second summand on the right hand of (12) side to obtain

$$
\zeta(1)=\int_{0}^{1} e^{\int_{t}^{1}-G_{\zeta} d \tau}\left(\left\langle\eta_{2}, \dot{\xi}_{1}\right\rangle-\left\langle\dot{\eta}_{1}, \xi_{2}\right\rangle-G_{\zeta}\left\langle\eta_{1}, \xi_{2}\right\rangle-d^{2} H_{t}\left(\mu_{1}, \mu_{2}\right)\right) d t .
$$

Since $c$ is critical observe, by Proposition 3.1, that

$$
\alpha\left(\mu_{i}(t)\right)=\zeta_{i}(t)-\left\langle y(t), \xi_{i}(t)\right\rangle=0
$$

for all $t \in[0,1]$ thus, in particular,

$$
d^{2} H_{t}\left(\mu_{1}, \mu_{2}\right)=\left\langle G_{\xi \xi} \xi_{1}, \xi_{2}\right\rangle+\left\langle G_{\xi \eta} \eta_{1}, \xi_{2}\right\rangle+\left\langle G_{\eta \xi} \xi_{1}, \eta_{2}\right\rangle+\left\langle G_{\eta \eta} \eta_{1}, \eta_{2}\right\rangle .
$$

The proposition now follows.

REMARK 5.6. $A(c)$ extends to a self-adjoint operator on $L^{2}\left([0,1], \mathbb{R}^{2 k}\right)$ with dense domain

$$
W_{c, M}^{1,2}=\left\{\gamma \in W^{1,2}\left([0,1] ; \mathbb{R}^{2 k}\right) \mid \gamma(0) \in \Lambda_{0}, \gamma(1) \in \Lambda_{l}\right\}
$$

In addition, $A(c)$ is injective if and only if $\Psi(1) \Lambda_{0}$ is transverse to $\Lambda_{1}$, where $\Psi(t)=$ $\Psi_{c, H}(t) \in \mathrm{Sp}(2 k)$ is given by

$$
\dot{\Psi}=-J_{0} S \Psi, \quad \Psi(0)=\mathbb{1} .
$$

In such a case we will say that $c$ is a nondegenerate critical point of $\Phi$.

REMARK 5.7. The contact isotopy $\varphi^{t}$, generated by $H$, linearised along the path $a=(c, z):[0,1] \rightarrow J^{1} \mathbb{R}^{k}$ and restricted to contact planes, gives rise to the path of linear conformal symplectomorphisms

$$
\left.d \varphi^{t}(a(0))\right|_{\xi_{a(0)}}:\left(\xi_{a(0)}, d \alpha_{a(0)}\right) \rightarrow\left(\xi_{a(t)}, d \alpha_{a(t)}\right) .
$$

This path may be naturally identified with the path $e^{\int_{0}^{\prime}-\frac{1}{2} G_{\zeta} d r} \Psi_{c, H}$, where $\Psi_{c . H}$ is given in Remark 5.6, via the canonical symplectomorphisms

$$
\left(\xi_{p}, d \alpha_{p}\right) \rightarrow\left(\mathbb{R}^{2 k}, \omega_{0}\right):(\xi, \eta,\langle y, \xi\rangle) \mapsto(\xi, \eta),
$$

where $p=(x, y, z)$. 
REMARK 5.8. Identify $T^{*} M$ with $T M$ using the induced inner product. The tangent space to $T M^{\perp}$ at $p=(x, y)$ is given by

$$
T_{p}\left(T M^{\perp}\right)=\left\{(\xi, \eta) \in \mathbb{R}^{2 k}: \xi \in T_{x} M,(d \Pi(x) \xi) y+\Pi(x) \eta=0\right\},
$$

where $\Pi(x) \in \mathbb{R}^{k \times k}$ denotes the orthogonal projection onto $T_{x} M$. Note that defining $I I_{p}: T_{x} M \rightarrow \mathbb{R}$ by $I_{p}(\xi)=\frac{1}{2}\langle(d \Pi(x) \xi) y, \xi\rangle$ gives a quadratic form on $T_{x} M$, the second fundamental form of $M$ at $x$ along the normal vector $y$.

REMARK 5.9. Let $G:[0,1] \times J^{1} \mathbb{R}^{k} \rightarrow \mathbb{R}$ be the time-dependent Hamiltonian given by

$$
G(t, \xi, \eta, \zeta)=\frac{1}{2}\left\langle G_{\xi \xi}(t) \xi, \xi\right\rangle+\left\langle G_{\eta \xi}(t) \xi, \eta\right\rangle+\frac{1}{2}\left\langle G_{\eta \eta}(t) \eta, \eta\right\rangle+G_{\zeta}(t) \zeta .
$$

We call such Hamiltonians, where $G_{\xi \xi}(t), G_{\eta \xi}(t), G_{\eta \eta}(t) \in \mathbb{R}^{k \times k}$ with $G_{\xi \xi}(t), G_{\eta \eta}(t)$ symmetric and $G_{\xi}(t) \in \mathbb{R}$, quadratic, and define $G_{\xi \eta}(t)=G_{\eta \xi}(t)^{T}$. Define the functional $\Phi_{c}=\Phi_{c, H, M}: \mathscr{C}_{c} \rightarrow \mathbb{R}$ by

$$
\Phi_{c}(\gamma)=\int_{0}^{1}(\langle\eta, \dot{\xi}\rangle-G(t, \xi, \eta, \zeta)) d t+I I_{c(1)}(\xi(1)),
$$

where $\zeta:[0,1] \rightarrow \mathbb{R}$ is the unique solution of the initial value problem

$$
\dot{\zeta}(t)=\langle\eta(t), \dot{\xi}(t)\rangle-G(t, \xi(t), \eta(t), \zeta(t)), \quad \zeta(0)=0 .
$$

Notice that if $c$ is a nondegenerate critical point of $\Phi$, then 0 is the unique critical point of $\Phi_{c}$, also nondegenerate, and in this case the above proposition shows that the second variation of $\Phi$ at $c$ agrees with the second variation of $\Phi_{c}$.

REMARK 5.10. A quadratic Hamiltonian $G:[0,1] \times J^{1} \mathbb{R}^{k} \rightarrow \mathbb{R}$ as above generates the contact isotopy

$$
\begin{aligned}
\left(\begin{array}{c}
\xi(t) \\
\eta(t)
\end{array}\right) & =e^{\int_{0}^{t}-\frac{1}{2} G_{\xi} d \tau} \Psi_{G}(t)\left(\begin{array}{c}
\xi_{0} \\
\eta_{0}
\end{array}\right), \\
\zeta(t) & =e^{\int_{0}^{t}-G_{\xi} d \tau} \zeta_{0}+\frac{1}{2} \int_{0}^{t} e^{\int_{s}^{t}-G_{\zeta} d \tau}\left(\left\langle G_{\eta \eta} \eta, \eta\right\rangle-\left\langle G_{\xi \xi} \xi, \xi\right\rangle\right) d s,
\end{aligned}
$$

where $\Psi_{G}:[0,1] \rightarrow \mathrm{Sp}(2 k)$ is given by

$$
\dot{\Psi}_{G}=-J_{0} S_{G} \Psi_{G}, \quad \Psi_{G}(0)=\mathbb{1} .
$$

Conversely, given any smooth family of symplectic matrices $\Psi:[0,1] \rightarrow \operatorname{Sp}(2 k)$ and any smooth positive function $a:[0,1] \rightarrow \mathbb{R}_{>0}$ there exists a contact isotopy of the form

$$
\left(\begin{array}{l}
\xi(t) \\
\eta(t)
\end{array}\right)=a(t) \Psi(t)\left(\begin{array}{l}
\xi_{0} \\
\eta_{0}
\end{array}\right), \quad \zeta(t)=a(t)^{2} \zeta_{0}+Q\left(t, \xi_{0}, \eta_{0}\right),
$$

where $\Psi=\Psi_{G}$ for some quadratic Hamiltonian $\mathrm{G}$ and $Q(t, \cdot, \cdot)$ is quadratic. We call such contact isotopies pseudo-linear. 


\section{Discrete variational families.}

In this section we construct a family of finite dimensional variational families $\left(\mathscr{P}^{N}, \Phi^{N}\right)$ for our Legendrian submanifold $\psi^{1}\left(M_{0} \times\{0\}\right) \subset J^{1} M$. This is done in two stages. The first stage is to consider the special case when $M$ is $\mathbb{R}^{n}$ and the contactomorphism $\psi^{1}$ is $C^{1}$-close to the identity. The second stage is the general case. Alternative constructions are given by Chekanov [5] and Théret [16]. The corresponding construction in the case of cotangent bundles was first carried out by Laudenbach and Sikorav [11].

6.1. Generating functions of type $V$. When $M$ is $\mathbb{R}^{n}$ and the contactomorphism is $C^{1}$-close to the identity it turns out that the contactomorphism is generated by a function defined on $\mathbb{R}^{n+1}$. We explain this presently.

Let $\varphi: J^{1} \mathbb{R}^{k} \rightarrow J^{1} \mathbb{R}^{k}$ be a contactomorphism and denote it as follows:

$$
\left(x_{0}, y_{0}, z_{0}\right) \mapsto\left(x_{1}, y_{1}, z_{1}\right)=\left(u\left(x_{0}, y_{0}, z_{0}\right), v\left(x_{0}, y_{0}, z_{0}\right), w\left(x_{0}, y_{0}, z_{0}\right)\right) .
$$

A generating function of type $V$ for $\varphi$ is a function $V: \mathbb{R}^{2 k+1} \rightarrow \mathbb{R}$ such that

$$
\begin{aligned}
& x_{1}-x_{0}=\frac{\partial V}{\partial y}\left(x_{0}, y_{1}, z_{0}\right), \\
& y_{1}-y_{0}=-\frac{\partial V}{\partial x}\left(x_{0}, y_{1}, z_{0}\right)-y_{0} \frac{\partial V}{\partial z}\left(x_{0}, y_{1}, z_{0}\right), \\
& z_{1}-z_{0}=\left\langle y_{1}, x_{1}-x_{0}\right\rangle-V\left(x_{0}, y_{1}, z_{0}\right)
\end{aligned}
$$

if and only if $\left(x_{1}, y_{1}, z_{1}\right)=\varphi\left(x_{0}, y_{0}, z_{0}\right)$. This generalises the notion of a generating function of type $V$, as defined in [12], to the contact setting. In case $\varphi$ admits a generating function of type $V$ note that $\varphi$ has compact support (that is, $\varphi$ is equal to the identity outside a compact set) if and only if $V$ has.

PROPOSITION 6.1. (i) Every contactomorphism $\varphi: J^{1} \mathbb{R}^{k} \rightarrow J^{1} \mathbb{R}^{k}$ which is sufficiently close to the identity in the $C^{1}$-topology admits a unique generating function of type $V$.

(ii) For each smooth function $V: \mathbb{R}^{2 k+1} \rightarrow \mathbb{R}$ having compact support and sufficiently small first and second derivatives there exists a unique contactomorphism $\varphi: J^{1} \mathbb{R}^{k} \rightarrow J^{1} \mathbb{R}^{k}$ such that $V$ is a generating function of type $V$ for $\varphi$.

PROOF. For (i), the assumption on $\varphi$ ensures that the map

$$
\mathbb{R}^{2 k+1} \rightarrow \mathbb{R}^{2 k+1}:\left(x_{0}, y_{0}, z_{0}\right) \mapsto\left(x_{0}, v\left(x_{0}, y_{0}, z_{0}\right), z_{0}\right)
$$


has a global inverse. Thus there exists a map $f: \mathbb{R}^{2 k+1} \rightarrow \mathbb{R}^{k}$ such that

$$
y_{0}=f\left(x_{0}, v\left(x_{0}, y_{0}, z_{0}\right), z_{0}\right) .
$$

Also, since $\varphi:\left(x_{0}, y_{0}, z_{0}\right) \mapsto\left(x_{1}, y_{1}, z_{1}\right)$ is a contactomorphism, we have

$$
d z_{1}-\sum y_{1 i} d x_{1 i}=e^{g}\left(d z_{0}-\sum y_{0 i} d x_{0 i}\right)
$$

for some function $g: \mathbb{R}^{2 k+1} \rightarrow \mathbb{R}$. Thus, using the notation set up earlier, the chain rule gives

$$
\begin{aligned}
d z_{1}-\sum y_{1 i} d x_{1 i}= & \sum\left(\partial_{x_{0 i}} w-\left\langle y_{1}, \partial_{x_{0 i}} u\right\rangle\right) d x_{0 i} \\
& +\sum\left(\partial_{y_{0 i}} w-\left\langle y_{1}, \partial_{y_{0 i}} u\right\rangle\right) d y_{0 i}+\left(\partial_{z_{0}} w-\left\langle y_{1}, \partial_{z_{0}} u\right\rangle\right) d z_{0}
\end{aligned}
$$

Comparing with (16) we find that

$$
\partial_{y_{0}} w-\left(\partial_{y_{0}} u\right)^{T} y_{1}=0
$$

and

$$
\partial_{x_{0}} w-\left(\partial_{x_{0}} u\right)^{T} y_{1}=-\left(\partial_{z_{0}} w-\left\langle y_{1}, \partial_{z_{0}} u\right\rangle\right) y_{0}
$$

We claim that $V: \mathbb{R}^{2 n+1} \rightarrow \mathbb{R}$ defined by

$$
\begin{aligned}
V\left(x_{0}, y_{1}, z_{0}\right): & =\left\langle y_{1}, x_{1}-x_{0}\right\rangle-z_{1}+z_{0} \\
& =\left\langle y_{1}, u\left(x_{0}, f\left(x_{0}, y_{1}, z_{0}\right), z_{0}\right)-x_{0}\right\rangle-w\left(x_{0}, f\left(x_{0}, y_{1}, z_{0}\right), z_{0}\right)+z_{0}
\end{aligned}
$$

satisfies (15).

This claim is proved by a straightforward calculation, differentiating (19) and using the identities (17) and (18). The uniqueness of generating functions of type $V$ is clear.

For (ii), let $V: \mathbb{R}^{2 k+1} \rightarrow \mathbb{R}$ be a smooth function satisfying the hypotheses. We construct $\varphi$ as follows. Let $\mu: \mathbb{R}^{2 k+1} \rightarrow \mathbb{R}^{2 k+1}$ be the map defined by

$$
\left(x_{0}, y_{1}, z_{0}\right) \mapsto\left(x_{0},\left(1-\partial_{z_{0}} V\left(x_{0}, y_{1}, z_{0}\right)\right)^{-1}\left(y_{1}+\partial_{x_{0}} V\left(x_{0}, y_{1}, z_{0}\right)\right), z_{0}\right)
$$

and $v: \mathbb{R}^{2 k+1} \rightarrow \mathbb{R}^{2 k+1}$ be the map defined by

$$
\left(x_{0}, y_{1}, z_{0}\right) \mapsto\left(x_{0}+\partial_{y_{1}} V, y_{1}, z_{0}+\left\langle y_{1}, \partial_{y_{1}} V\right\rangle-V\right)
$$

The hypotheses on $V$ ensure that $\mu$ and $\nu$ are diffeomorphisms. We define $\varphi$ to be the composition $\nu \circ \mu^{-1}$. That $\varphi$ is a contactomorphism is seen directly as follows. Expand the formula for the contact 1 -form $\alpha=d z_{1}-\sum y_{1 i} d x_{1 i}$. After cancellations this becomes

$$
d z_{1}-\sum y_{1 i} d x_{1 i}=\left(1-\partial_{z_{0}} V\right) d z_{0}-\sum\left(\partial_{x_{0 i}} V+y_{1 i}\right) d x_{0 i}
$$

From the definition of the function $y_{0}$ we have the equality

$$
\partial_{x_{0}} V+y_{1}=\left(1-\partial_{z 0} V\right) y_{0}
$$

hence $\varphi$ is a contactomorphism as required. 
The pseudo-linear case. When $\varphi$ is the time- $t_{1}$ map of a contact isotopy generated by a quadratic Hamiltonian $G$ the pair $\left(\xi_{1}, \eta_{1}\right)$ is given in terms of $\left(\xi_{0}, \eta_{0}\right)$ by

$$
\left(\begin{array}{l}
\xi_{1} \\
\eta_{1}
\end{array}\right)=a \Psi\left(\begin{array}{l}
\xi_{0} \\
\eta_{0}
\end{array}\right)
$$

where $a>0$ and

$$
\Psi=\left(\begin{array}{ll}
A & B \\
C & D
\end{array}\right) \in \operatorname{Sp}(2 k)
$$

are as given in Remark 5.10. In this case the condition of $\varphi$ being $C^{1}$-close to the identity is replaced by requirement that

$$
\operatorname{det}(D) \neq 0
$$

which holds for $t_{1}>0$ sufficiently small. Again, we say a generating function $\mathbb{R}^{2 k+1} \rightarrow \mathbb{R}:(\xi, \eta, \zeta) \mapsto W(\xi, \eta, \zeta)$ is quadratic if it is quadratic in $\xi$ and $\eta$ and linear in $\zeta$. A simple modification of the proof of Proposition 6.1 now gives:

PROPOSITION 6.2. Every contactomorphism $\varphi: J^{1} \mathbb{R}^{k} \rightarrow J^{1} \mathbb{R}^{k}$ generated by a quadratic Hamiltonian with (21) holding admits a unique quadratic generating function of type $V$.

Conversely, for each function $W=W(\xi, \eta, \zeta)$ on $\mathbb{R}^{2 k+1}$, quadratic in $\xi$ and $\eta$ and linear in $\zeta$, satisfying

$$
1-\partial_{\zeta} W \neq 0, \quad \operatorname{det}\left(\mathbb{1}+\partial_{\xi \eta}^{2} W\right) \neq 0,
$$

there exists a unique pseudo-linear contactomorphism $\varphi: J^{1} \mathbb{R}^{k} \rightarrow J^{1} \mathbb{R}^{k}$ such that $W$ is a generating function of type $V$ for $\varphi$.

We now give an explicit formula for the quadratic generating function of the pseudolinear contactomorphism $\varphi$ above. Observe from (20) that when condition (21) holds

$$
\begin{aligned}
\xi_{1}-\xi_{0} & =(a A-\mathbb{1}) \xi_{0}+a B \eta_{0} \\
& =(a A-\mathbb{1}) \xi_{0}+B D^{-1} \eta_{1}-a B D^{-1} C \xi_{0} \\
& =\left(a A-a B D^{-1} C-\mathbb{1}\right) \xi_{0}+B D^{-1} \eta_{1} \\
& =\left(a\left(D^{-1}\right)^{T}-\mathbb{1}\right) \xi_{0}+B D^{-1} \eta_{1} .
\end{aligned}
$$

Here the last equality follows from the fact that $\Psi \in S p(2 k)$. Also note that

$$
\begin{aligned}
\eta_{1}-\eta_{0} & =\eta_{1}-a^{-1} D^{-1} \eta_{1}+D^{-1} C \xi_{0} \\
& =D^{-1} C \xi_{0}+\left(\mathbb{1}-a D^{-1}\right) \eta_{1}+\left(a-a^{-1}\right) D^{-1} \eta_{1} \\
& =D^{-1} C \xi_{0}+\left(\mathbb{1}-a D^{-1}\right) \eta_{1}+\left(a-a^{-1}\right) D^{-1}\left(a C \xi_{0}+a D \eta_{0}\right) \\
& =a^{2} D^{-1} C \xi_{0}+\left(\mathbb{1}-a D^{-1}\right) \eta_{1}+\left(a^{2}-1\right) \eta_{0} .
\end{aligned}
$$


It follows that the generating function of type $V$ for $\varphi$ is given by

$$
\begin{aligned}
W\left(\xi_{0}, \eta_{1}, \zeta_{0}\right)= & -\frac{1}{2}\left\langle a^{2} D^{-1} C \xi_{0}, \xi_{0}\right\rangle+\left\langle\left(a\left(D^{-1}\right)^{T}-1\right) \xi_{0}, \eta_{1}\right\rangle \\
& +\frac{1}{2}\left\langle B D^{-1} \eta_{1}, \eta_{1}\right\rangle+\left(1-a^{2}\right) \zeta_{0} .
\end{aligned}
$$

6.2. Discrete-time variational theory. We consider the general case where $M$ is an arbitrary closed manifold embedded in $\mathbb{R}^{k}$ and $\psi^{t}: J^{1} M \rightarrow J^{1} M$ is a given contact isotopy. We assume that $\psi^{t}$ is generated by the contact Hamiltonian $\bar{H}:[0,1] \times$ $J^{1} M \rightarrow \mathbb{R}$ and that $H:[0,1] \times J^{1} \mathbb{R}^{k} \rightarrow \mathbb{R}$ is some function which satisfies (6). It follows that $H$ will not have compact support. Hence we multiply $H$ by a smooth cutoff function and assume, by abuse of notation, that $H$ is equal to zero outside of a large compact set containing $\bigcup_{t \in[0,1]} \varphi^{t}(M)$, where $\varphi^{t}$ denotes the contact isotopy generated by $H$, and $M$ the set $M \times\{0\} \times\{0\} \subset J^{1} \mathbb{R}^{k}$.

We now construct a family of finite dimensional variational families generating the Legendrian submanifold $\psi^{1}\left(M_{0} \times\{0\}\right)$, by discretising the time variable. Pick an integer $N$ and define

$$
\varphi_{j}^{j+1}=\varphi^{(j+1) / N} \circ\left(\varphi^{j / N}\right)^{-1}
$$

for $j=0, \ldots, N-1$. Then

$$
\varphi^{1}=\varphi_{N-1}^{N} \circ \varphi_{N-2}^{N-1} \circ \cdots \circ \varphi_{0}^{1}
$$

and for $N$ sufficiently large each $\varphi_{j}^{j+1}$ satisfies the hypotheses of Proposition 6.1 (i). Hence for each $j$ there exists a function $V_{j}: \mathbb{R}^{2 k+1} \rightarrow \mathbb{R}$ such that

$$
\begin{aligned}
x_{j+1}-x_{j} & =\frac{\partial V_{j}}{\partial y}, \\
y_{j+1}-y_{j} & =-\frac{\partial V_{j}}{\partial x}-y_{j} \frac{\partial V_{j}}{\partial z}, \\
z_{j+1}-z_{j} & =\left\langle y_{j+1}, x_{j+1}-x_{j}\right\rangle-V_{j}
\end{aligned}
$$

if and only if $\left(x_{j+1}, y_{j+1}, z_{j+1}\right)=\varphi_{j}^{j+1}\left(x_{j}, y_{j}, z_{j}\right)$.

Now define, by analogy with the continuous-time case, the space

$$
\mathscr{P}^{N}=\mathscr{P}_{\mathbb{R}^{k}, M}^{N}=\left\{c=\left(x_{0}, \ldots, x_{N}, y_{1}, \ldots, y_{N}\right) \in \mathbb{R}^{(2 N+1) k} \mid x_{N} \in M\right\}
$$

of discrete paths in $\mathbb{R}^{2 k}$, and to each $c \in \mathscr{P}^{N}$ associate a sequence $\left(z_{0}, \ldots, z_{N}\right)$ given by the iterative rule

$$
z_{0}=0, \quad z_{j}=\left\langle y_{j}, x_{j}-x_{j-1}\right\rangle-V_{j-1}\left(x_{j-1}, y_{j}, z_{j-1}\right)+z_{j-1}, \quad j>0 .
$$

Let $\pi: \mathscr{P}^{N} \rightarrow M$ denote the projection $c \mapsto x_{N}$. The discrete action functional $\Phi^{N}=\Phi_{H . M}^{N}: \mathscr{P}^{N} \rightarrow \mathbb{R}$ can now be defined by

$$
\Phi^{N}(c)=z_{N}=\sum_{j=1}^{N}\left(\left\langle y_{j}, x_{j}-x_{j-1}\right\rangle-V_{j-1}\left(x_{j-1}, y_{j}, z_{j-1}\right)\right) .
$$


PROPOSITION 6.3. $\left(\mathscr{P}^{N}, \Phi^{N}\right)$ is a variational family for the Legendrian submanifold $\psi^{1}\left(M_{0} \times\{0\}\right) \subset J^{1} M$.

Proposition 6.3 follows from Lemma 6.4 .

LEMMA 6.4. There is a one-to-one correspondence between the set of fibre critical points of $\Phi^{N}$ and solutions of (24) (for $j=0, \ldots, N-1$ ) with $y_{0}=z_{0}=0, x_{N} \in M$.

PROOF. The partial derivatives of $\Phi^{N}$ are

$$
\begin{aligned}
& \frac{\partial \Phi^{N}}{\partial x_{j}}=\left(1-\partial_{z} V_{N-1}\right) \cdots\left(1-\partial_{z} V_{j+1}\right)\left(y_{j}-y_{j+1}-\partial_{x} V_{j}-y_{j} \partial_{z} V_{j}\right), \\
& \frac{\partial \Phi^{N}}{\partial y_{j+1}}=\left(1-\partial_{z} V_{N-1}\right) \cdots\left(1-\partial_{z} V_{j+1}\right)\left(x_{j+1}-x_{j}-\partial_{y} V_{j}\right)
\end{aligned}
$$

for $j=0, \ldots, N-1$ (on defining $y_{0}=0$ ). Since $1-\partial_{z} V_{l}$ is nonzero for each $l$, this proves the lemma.

As before, $c \in \mathscr{P}^{N}$ is a critical point of $\Phi^{N}$ if and only if $(c, z)$ is a solution of Hamilton's discrete equations (24) and $c_{N}=\left(x_{N}, y_{N}\right) \in T M^{\perp}$. In particular, $c$ is a critical point of $\Phi^{N}$ if and only if it is obtained by sampling a path $c \in$ crit $\Phi$.

REMARK 6.5. In applications it will be useful to note the following. Let $x=x_{N}$ and $\xi=\left(x_{0}, \ldots, x_{N-1}, y_{1}, \ldots, y_{N}\right)$ denote the base and fibre coordinates respectively of $\mathscr{P}^{N}=M \times \mathbb{R}^{2 m}$ where $m=N k$. Then $\Phi^{N}$ can be written in the form

$$
\Phi^{N}(x, \xi)=\frac{1}{2}\langle P \xi, \xi\rangle+W(x, \xi),
$$

where $P \in \mathbb{R}^{2 m \times 2 m}$ is a nondegenerate symmetric matrix of signature zero and $W$ is given by

$$
W(x, \xi)=\left\langle x, y_{N}\right\rangle-\sum_{j=1}^{N} V_{j-1}\left(x_{j-1}, y_{j}, z_{j-1}\right) .
$$

Also, the gradient of $W$ with respect to $\xi, \partial_{\xi} W$, is bounded.

REMARK 6.6. Suppose $L \subset J^{1} M$ is a Legendrian submanifold given by a variational family $\left(E=M \times \mathbb{R}^{l}, S\right.$ ). Then $\psi^{1}(L)$ is also given by a variational family. This is seen as follows.

Choose any function $\widetilde{S}: \widetilde{E} \rightarrow \mathbb{R}$, where $\widetilde{E}=\mathbb{R}^{k} \times \mathbb{R}^{l}$, satisfying $\left.\widetilde{S}\right|_{E}=S$, and define the space

$$
\widetilde{\mathscr{P}}^{N}=\left\{(\xi, c) \in \mathbb{R}^{l} \times \mathbb{R}^{(2 N+1) k} \mid c \in \mathscr{P}^{N}\right\}
$$


of augmented discrete paths. Now define the generalised discrete action functional $\widetilde{\Phi}^{N}: \widetilde{\mathscr{P}}^{N} \rightarrow \mathbb{R}$ by

$$
\widetilde{\Phi}^{N}(\xi, c)=z_{N}=\sum_{j=1}^{N}\left(\left\langle y_{j}, x_{j}-x_{j-1}\right\rangle-V_{j-1}\left(x_{j-1}, y_{j}, z_{j-1}\right)\right)+z_{0},
$$

where

$$
z_{0}=\tilde{S}\left(x_{0}, \xi\right), \quad z_{j}=\left\langle y_{j}, x_{j}-x_{j-1}\right\rangle-V_{j-1}\left(x_{j-1}, y_{j}, z_{j-1}\right)+z_{j-1}, \quad j>0 .
$$

It is now easy to check that $\left(\widetilde{\mathscr{P}}^{N}, \widetilde{\Phi}^{N}\right)$ is a variational family for $\psi^{1}(L)$.

REMARK 6.7. An alternative and more explicit way of constructing a discrete action functional is by setting

$$
V_{j}\left(x_{j}, y_{j+1}, z_{j}\right)=H\left(t_{j}, x_{j}, y_{j+1}, z_{j}\right)\left(t_{j+1}-t_{j}\right),
$$

where $t_{j}=j / N$ and $N$ is sufficiently large so that the hypotheses of Proposition 6.1 (ii) hold for each $V_{j}$. A disadvantage of this method is that it does not generate the original Legendrian submanifold but one that is close to it. This is the approach used by Robbin and Salamon in the symplectic setting [14].

6.3. The second variation. We start with some notation. Let $c \in \mathscr{P}^{N}$ be a critical point of $\Phi^{N}$ and $\left\{z_{j}\right\}$ the associated sequence given by the iterative rule (25). For $j=0, \ldots, N-1$ define $W_{j, \xi \xi}, W_{j, \xi \eta}, W_{j, \eta \xi}, W_{j, \eta \eta} \in \mathbb{R}^{k \times k}$ and $W_{j, \zeta} \in \mathbb{R}$ by

$$
\begin{aligned}
W_{j, \xi \xi} & =\partial_{x x}^{2} V_{j}+\partial_{x z}^{2} V_{j} y_{j}^{T}+y_{j} \partial_{z x}^{2} V_{j}+\partial_{z z}^{2} V_{j} y_{j} y_{j}^{T}, \\
W_{j, \xi \eta} & =\partial_{x y}^{2} V_{j}+y_{j} \partial_{z y}^{2} V_{j}, \\
W_{j, \eta \xi} & =\partial_{y x}^{2} V_{j}+\partial_{y z}^{2} V_{j} y_{j}^{T}, \\
W_{j, \eta \eta} & =\partial_{y y}^{2} V_{j}, \\
W_{j, \zeta} & =\partial_{z} V_{j},
\end{aligned}
$$

where $V_{j}$ is evaluated at $\left(x_{j}, y_{j+1}, z_{j}\right)$. Note that $W_{j, \xi \xi}, W_{j, \eta \eta}$ are symmetric and $W_{j, \xi \eta}=W_{j, \eta \xi}^{T}$. Abbreviate $\alpha_{j}^{l}=\left(1-\partial_{z} V_{l}\right) \cdots\left(1-\partial_{z} V_{j}\right)$ for $l \geq j$, setting it to 1 otherwise. Denote by $W_{c}^{N}=W_{c, M}^{N}$ the tangent space of $\mathscr{P}^{N}$ at $c$ :

$$
W_{c}^{N}=\left\{\gamma=\left(\xi_{0}, \ldots, \xi_{N}, \eta_{1}, \ldots, \eta_{N}\right) \in \mathbb{R}^{(2 N+1) k} \mid \xi_{N} \in T_{x_{N}} M\right\} .
$$

We now give a formula for the second variation of $\Phi^{N}$ at $c$. As before, $\Pi(x) \in \mathbb{R}^{k \times k}$ denotes the orthogonal projection onto $T_{x} M$. 
PROPOSITION 6.8. The second derivative of $\Phi^{N}$ at a critical point $c$ is of the form

$$
d^{2} \Phi^{N}(c)\left(\gamma_{1}, \gamma_{2}\right)=\left\langle A^{N}(c) \gamma_{1}, \gamma_{2}\right\rangle
$$

where $\gamma_{1}, \gamma_{2} \in W_{c}^{N}$ and the second variation, $A^{N}(c): W_{c}^{N} \rightarrow W_{c}^{N}:(\xi, \eta) \mapsto(u, v)$, is given by the expressions

$$
\begin{aligned}
u_{j} & =\alpha_{j+1}^{N-1}\left(\eta_{j}-\eta_{j+1}-W_{j, \xi \xi} \xi_{j}-W_{j, \xi \eta} \eta_{j+1}-W_{j, \zeta} \eta_{j}\right) \\
v_{j+1} & =\alpha_{j+1}^{N-1}\left(\xi_{j+1}-\xi_{j}-W_{j, \eta \xi} \xi_{j}-W_{j, \eta \eta} \eta_{j+1}\right) \\
u_{N} & =\left(d \Pi\left(x_{N}\right) \xi_{N}\right) y_{N}+\Pi\left(x_{N}\right) \eta_{N}
\end{aligned}
$$

for $j=0, \ldots, N-1$, where $\eta_{0}=0$.

PROOF. Differentiate the formulae in the proof of Lemma 6.4.

REMARK 6.9. For $j=0, \ldots, N-1$ let $W_{j}: \mathbb{R}^{2 k+1} \rightarrow \mathbb{R}$ be the function defined by

$$
W_{j}\left(\xi_{j}, \eta_{j+1}, \zeta_{j}\right)=\frac{1}{2}\left\langle W_{j, \xi \xi} \xi_{j}, \xi_{j}\right\rangle+\left\langle W_{j, \eta \xi} \xi_{j}, \eta_{j+1}\right\rangle+\frac{1}{2}\left\langle W_{j, \eta \eta} \eta_{j+1}, \eta_{j+1}\right\rangle+W_{j, \zeta} \zeta_{j}
$$

Using these we may associate with the critical point $c$ the function $\Phi_{c}^{N}=\Phi_{c, H, M}^{N}: W_{c}^{N} \rightarrow$ $\mathbb{R}$ defined by

$$
\Phi_{c}^{N}(\gamma)=\sum_{j=1}^{N}\left(\left\langle\eta_{j}, \xi_{j}-\xi_{j-1}\right\rangle-W_{j-1}\left(\xi_{j-1}, \eta_{j}, \zeta_{j-1}\right)\right)+I_{c_{N}}\left(\xi_{N}\right),
$$

where $\zeta_{j}$ is given by the iterative rule

$$
\zeta_{0}=0, \quad \zeta_{j}=\left\langle\eta_{j}, \xi_{j}-\xi_{j-1}\right\rangle-W_{j-1}\left(\xi_{j-1}, \eta_{j}, \zeta_{j-1}\right)+\zeta_{j-1}, \quad j>0
$$

and where $I I_{c_{N}}$ denotes the second fundamental form. As in the continuous-time case, if $c$ is a nondegenerate critical point of $\Phi^{N}$, then 0 is the unique critical point of $\Phi_{c}^{N}$, also nondegenerate, and in this case Proposition 6.8 implies that the second variation of $\Phi^{N}$ at $c$ agrees with the second variation of $\Phi_{c}^{N}$.

Recall that in the continuous-time case given a critical point $c$ of $\Phi$ we can construct a functional $\Phi_{c}$ whose second variation agrees with the second variation of $\Phi$ at $c$ (see Remark 5.9). The following proposition shows how $\Phi_{c}^{N}$ and $\Phi_{c}$ are related.

PROPOSITION 6.10. $\Phi_{c}^{N}$ is the discretisation of $\Phi_{c}$.

PROOF. Let $(c, z):[0,1] \rightarrow J^{1} M$ be a solution of Hamilton's equations beginning in $L_{0} \times\{0\}$ and $\left(c^{N},\left\{z_{j}\right\}\right)$ the corresponding solution of Hamilton's discrete equations. 
Note that, by Remark 5.7, linearising Hamilton's equations for $H$ along $(c, z)$ and restricting to contact planes, which are canonically identified with $\mathbb{R}^{2 k}$, yields the equations

$$
\begin{aligned}
& \dot{\xi}=G_{\eta \xi} \xi+G_{\eta \eta} \eta, \\
& \dot{\eta}=-G_{\xi \xi} \xi-G_{\xi \eta} \eta-G_{\zeta} \eta .
\end{aligned}
$$

Similarly, linearising Hamilton's discrete equations along $\left(c^{N},\left\{z_{j}\right\}\right)$ and restricting to contact planes yields the equations

$$
\begin{aligned}
\xi_{j+1}-\xi_{j} & =W_{j, \eta \xi} \xi_{j}+W_{j, \eta \eta} \eta_{j+1}, \\
\eta_{j+1}-\eta_{j} & =-W_{j, \xi \xi} \xi_{j}-W_{j, \xi \eta} \eta_{j+1}-W_{j, \zeta} \eta_{j} .
\end{aligned}
$$

It follows that the $W_{j}$ s define a family of generating functions of type $V$ for the pseudo-linear contact isotopy generated by $G$ and the result now follows.

COROLLARY 6.11. $\left(\mathscr{P}^{N}, \Phi^{N}\right)$ is always transversal as a variational family.

PROOF. We need to show that, given $\gamma=\left(\xi_{0}, \ldots, \xi_{N}, \eta_{1}, \ldots, \eta_{N}\right) \in W_{c}^{N}$ satisfying $\xi_{N}=0$, if $d^{2} \Phi^{N}(c)\left(\gamma, \gamma^{\prime}\right)$ is 0 for all $\gamma^{\prime} \in W_{c}^{N}$, then $\gamma$ is identically 0 . This is seen as follows. Suppose $\gamma$ satisfies the hypotheses of the statement we are seeking to show, then

$$
\begin{aligned}
\xi_{j+1}-\xi_{j} & =W_{j, \eta \xi} \xi_{j}+W_{j, \eta \eta} \eta_{j+1}, \\
\eta_{j+1}-\eta_{j} & =-W_{j, \xi \xi} \xi_{j}-W_{j, \xi \eta} \eta_{j+1}-W_{j, \zeta} \eta_{j}
\end{aligned}
$$

for $j=1, \ldots, N-1$, and $\Pi\left(x_{N}\right) \eta_{N}=0$. Denoting by $A_{j}$ the conformal symplectic matrix associated to $W_{j}$, it follows that

$$
\left(\begin{array}{c}
\xi_{j+1} \\
\eta_{j+1}
\end{array}\right)=A_{j}\left(\begin{array}{l}
\xi_{j} \\
\eta_{j}
\end{array}\right)
$$

for $j=1, \ldots, N-1$, and that $\left(\xi_{N}, \eta_{N}\right)$ is in the symplectic complement of the coisotropic subspace $N_{c_{N}}=T_{c_{N}}\left(\left.T^{*} \mathbb{R}^{k}\right|_{M}\right)$. Since $\varphi^{t}$ preserves $\left.J^{1} \mathbb{R}^{k}\right|_{M}$, we have $A_{j} N_{c_{j}}=N_{c_{j+1}}$. It follows that $\left(\xi_{0}, 0\right)$ is in the symplectic complement of $N_{c_{0}}$ which implies that $\xi_{0}=0$ and hence $\gamma$ is identically 0 , as required.

COROLLARY 6.12. The second variation of $\Phi^{N}$ at a critical point $c$ is nondegenerate if and only if $\Psi_{c, H} \Lambda_{0}$ is transverse to $\Lambda_{1}=T_{c_{N}}\left(T M^{\perp}\right)$.

PROOF. This follows immediately from Corollary 6.11 , Corollary 4.6 and Remark 5.7 .

REMARK 6.13. If the variational family $(E, S)$, given in Remark 6.6, is transversal, then the variational family $\left(\widetilde{\mathscr{P}}^{N}, \widetilde{\Phi}^{N}\right)$ will also be transversal. This follows by arguing as in the proof of Corollary 6.11 and using Lemma 4.5. 


\section{The Maslov index}

In this section we define the Maslov index for the points of intersection of $\psi^{1}\left(M_{0} \times\{0\}\right)$ and $M_{0} \times \mathbb{R}$, assuming that the intersection is transversal. We begin by summarising the properties of the Maslov index for various spaces of paths that we will require. Proofs of these may be found in [13].

Let $\Lambda:[a, b] \rightarrow \mathscr{L}(k)$ be a path of Lagrangian subspaces and let $Z=(X, Y):[a, b]$ $\rightarrow \mathscr{L}\left(\mathbb{R}^{k}, \mathbb{R}^{2 k}\right)$ denote a choice of a frame for $\Lambda$. This means that $Z(t): \mathbb{R}^{k} \rightarrow \mathbb{R}^{2 k}$ is an injective linear map such that

$$
\Lambda(t)=\operatorname{Im} Z(t), \quad X(t)^{T} Y(t)=Y(t)^{T} X(t)
$$

for all $t$. Also, let $V \in \mathscr{L}(k)$ be a fixed Lagrangian subspace. A crossing for the path $\Lambda$ is a number $t \in[a, b]$ such that $\Lambda(t)$ intersects $V$ nontrivially. At a crossing $t$ the crossing form is defined to be the quadratic form

$$
\Gamma(\Lambda, V, t): \Lambda(t) \cap V \rightarrow \mathbb{R}
$$

given by

$$
v \mapsto\langle X(t) u, \dot{Y}(t) u\rangle-\langle Y(t) u, \dot{X}(t) u\rangle,
$$

where $v=Z(t) u$. A crossing $t$ is said to be regular if $\Gamma(\Lambda, V, t)$ is nonsingular. When the path $\Lambda$ has only regular crossings the Maslov index, $\mu(\Lambda, V)$, is defined by

$$
\mu(\Lambda, V)=\frac{1}{2} \operatorname{sign} \Gamma(\Lambda, V, a)+\sum_{a<t<b} \operatorname{sign} \Gamma(\Lambda, V, t)+\frac{1}{2} \operatorname{sign} \Gamma(\Lambda, V, b),
$$

where the sum is taken over all crossings $t$. By perturbing, keeping endpoints fixed, this definition extends to give a well defined Maslov index for all paths. The integers $k_{a}$ and $k_{b}$ are defined by

$$
k_{a}=\operatorname{dim} \Lambda(a) \cap V, \quad k_{b}=\operatorname{dim} \Lambda(b) \cap V .
$$

We now state some properties of the Maslov index.

(Integrality.) The integers $\mu, k_{a}$ and $k_{b}$ are related by

$$
\mu+\left(k_{a}-k_{b}\right) / 2 \in \mathbb{Z} .
$$

(Product.) Under the natural identification of $\mathscr{L}(k) \times \mathscr{L}\left(k^{\prime}\right)$ as a submanifold of $\mathscr{L}\left(k+k^{\prime}\right)$

$$
\mu\left(\Lambda \oplus \Lambda^{\prime}, V \oplus V^{\prime}\right)=\mu(\Lambda, V)+\mu\left(\Lambda^{\prime}, V^{\prime}\right)
$$


(Localisation.) For the path $\Lambda$ given by the frame $t \mapsto(\mathbb{1}, A(t))$ and $V$ the subspace $\mathbb{R}^{k} \times\{0\}$ the Maslov index is given by

$$
\mu(\Lambda, V)=\frac{1}{2} \operatorname{sign} A(b)-\frac{1}{2} \operatorname{sign} A(a) .
$$

(Zero.) If $\Lambda(t) \cap V$ has constant dimension for all $t$ then $\mu(\Lambda, V)=0$.

For a pair of Lagrangian paths $\Lambda, \Lambda^{\prime}:[a, b] \rightarrow \mathscr{L}(k)$ the relative crossing form $\Gamma\left(\Lambda, \Lambda^{\prime}, t\right)$ on $\Lambda(t) \cap \Lambda(t)^{\prime}$ is defined by

$$
\Gamma\left(\Lambda, \Lambda^{\prime}, t\right)=\Gamma\left(\Lambda, \Lambda^{\prime}(t), t\right)-\Gamma\left(\Lambda(t), \Lambda^{\prime}, t\right) .
$$

When the pair $\Lambda, \Lambda^{\prime}$ have only regular crossings the relative Maslov index, $\mu\left(\Lambda, \Lambda^{\prime}\right)$, is defined by

$$
\mu\left(\Lambda, \Lambda^{\prime}\right)=\frac{1}{2} \operatorname{sign} \Gamma\left(\Lambda, \Lambda^{\prime}, a\right)+\sum_{a<t<b} \operatorname{sign} \Gamma\left(\Lambda, \Lambda^{\prime}, t\right)+\frac{1}{2} \operatorname{sign} \Gamma\left(\Lambda, \Lambda^{\prime}, b\right) .
$$

The relative Maslov index has the following property.

(Naturality.) For a Lagrangian pair $\Lambda, \Lambda^{\prime}$ and a symplectic path $\Psi$

$$
\mu\left(\Psi \Lambda, \Psi \Lambda^{\prime}\right)=\mu\left(\Lambda, \Lambda^{\prime}\right)
$$

Finally, for a path of symplectic matrices $\Psi:[a, b] \rightarrow \operatorname{Sp}(2 k)$ the Maslov index, $\mu(\Psi)$, is defined by

$$
\mu(\Psi)=\mu(\Psi V, V)
$$

where $V=\mathbb{R}^{k} \times\{0\}$. (Note that this definition differs from the one given in [13] since here the Maslov index is defined with respect to the horizontal and there with respect to the vertical.)

The following property of the Maslov index for symplectic paths will be used.

(Homotopy.) Two symplectic paths $\Psi, \Psi^{\prime}$ with $\Psi(a)=\Psi^{\prime}(a)$ and $\Psi(b), \Psi^{\prime}(b) \epsilon$ $\mathrm{Sp}_{0}(2 k)$ are homotopic within this class if and only if they have the same Maslov index, where $\mathrm{Sp}_{0}(2 k)$ denote the set of matrices $\Psi \in \mathrm{Sp}(2 k)$ such that $\Psi V$ transverse to $V$.

We now define the Maslov index for the points of intersection of $\psi^{1}\left(M_{0} \times\{0\}\right)$ and $M_{0} \times \mathbb{R}$, or equivalently, the critical points of $\Phi_{\bar{H}}$.

Let $\bar{c} \in \mathscr{P}_{M}$ be a critical point of $\Phi_{\bar{H}}$ and denote by $c=(x, y) \in \mathscr{P}$ its unique lift to a critical point of $\Phi$ (see Remark 5.4). Induced by $c$ is the path of symplectic matrices

$$
\Psi_{c, H}:[0,1] \rightarrow \operatorname{Sp}(2 k)
$$


(see Remarks 5.6 and 5.7) and this reduces, via symplectic reduction of the coisotropic distribution $T\left(\left.T^{*} \mathbb{R}^{k}\right|_{M}\right)$, to the path of symplectic linear transformations

$$
\Psi_{\bar{c}, \bar{H}}(t):\left(T_{\bar{c}(0)}\left(T^{*} M\right),-d \lambda_{\operatorname{can} \bar{c}(0)}\right) \longrightarrow\left(T_{\bar{c}(t)}\left(T^{*} M\right),-d \lambda_{\operatorname{can} \bar{c}(t)}\right) .
$$

We define the Maslov index, $\mu(\bar{c}, \bar{H})$, by

$$
\mu(\bar{c}, \bar{H})=\mu\left(\Psi_{\bar{c}, \bar{H}} \bar{\Lambda}_{0}, \bar{\Lambda}_{1}\right),
$$

where $\bar{\Lambda}_{0}=T_{x(0)} M$ and $\bar{\Lambda}_{1}$ is the path obtained by reducing

$$
\Lambda_{1}(t)=\left\{(\xi, \eta) \in \mathbb{R}^{2 k}: \xi \in T_{x(t)} M, \Pi(x(t))(d \Pi(x(t)) \xi) y(t)+\Pi(x(t)) \eta=0\right\} .
$$

Here the right hand side of (27) is defined by choosing a unitary trivialisation of the vector bundle $\bar{c}^{*} T\left(T^{*} M\right)$. By the naturality property this is independent of the trivialisation chosen. By the integrality property

$$
\mu(\bar{c}, \bar{H}) \in n / 2+\mathbb{Z} .
$$

If we assume that the embedding $M \hookrightarrow \mathbb{R}^{k}$ is an isometry, then this definition is independent of the choice of embedding. Indeed, the induced Riemannian structure on $T^{*} M$, via the embedding $M \hookrightarrow \mathbb{R}^{k}$, induces a splitting of $T_{p}\left(T^{*} M\right)$ into a horizontal and a vertical space:

$$
T_{p}\left(T^{*} M\right)=H_{p} \oplus V_{p}
$$

given by

$$
\begin{aligned}
H_{p} & =\left\{(\xi,(d \Pi(x) \xi) y) \in \mathbb{R}^{2 k}: \xi \in T_{x} M\right\}, \\
V_{p} & =\left\{(0, \eta) \in \mathbb{R}^{2 k}: \eta \in T_{x} M\right\},
\end{aligned}
$$

where $p=(x, y)$. In this notation $\bar{\Lambda}_{1}(t)$ is just the horizontal subspace $H_{\bar{c}(t)}$.

LEMMA 7.1. Let $\bar{c} \in \mathscr{P}_{M}$ and $c \in \mathscr{P}$ be critical point of $\Phi_{\bar{H}}$ and $\Phi$ respectively and related as above. Then there is an equality

$$
\mu(\bar{c}, \bar{H})=\mu\left(\Psi_{c . H} \Lambda_{0}, \Lambda_{1}(1)\right)+\frac{1}{2} \operatorname{sign} I I_{c(1)},
$$

where $\Lambda_{1}:[0,1] \rightarrow \mathscr{L}(k)$ is given by (28).

The proof of this lemma uses the following fact about symplectic reductions.

LEMMA 7.2. Let $\Lambda_{0}, \Lambda_{1}:[0,1] \rightarrow \mathscr{L}(k)$ be a pair of Lagrangian paths and $N \subset$ $\mathbb{R}^{2 k}$ a coisotropic subspace such that

$$
\Lambda_{0}(t) \cap N^{\omega}=\{0\}, \quad \Lambda_{1}(t) \subset N .
$$


Then the Maslov index of the pair $\left(\Lambda_{0}, \Lambda_{1}\right)$ agrees with the Maslov index of the reduced $\operatorname{pair}\left(\bar{\Lambda}_{0}, \bar{\Lambda}_{1}\right)$

$$
\mu\left(\Lambda_{0}, \Lambda_{1}\right)=\mu\left(\bar{\Lambda}_{0}, \bar{\Lambda}_{1}\right)
$$

PROOF. By choosing a basis for the isotropic subspace $N^{\omega}$ and then extending it to a basis of the Lagrangian subspace $\Lambda_{1}(t)$ we can assume that

$$
\begin{aligned}
N & =\mathbb{R}^{n} \times\{0\} \times \mathbb{R}^{n} \times \mathbb{R}^{k-n}, \\
\Lambda_{1} & =\mathbb{R}^{n} \times\{0\} \times\{0\} \times \mathbb{R}^{k-n}
\end{aligned}
$$

for all $t$. Hence it is sufficient to check that the assertion of the proposition holds when only $\Lambda_{0}$ is allowed to vary and $\Lambda_{1}$ and $N$ are as above. Since $\Lambda_{0}(t) \cap N^{\omega}=$ $\{0\}$ it follows that we may choose a Lagrangian frame for $\Lambda_{0}$ of the form $Z=$ $(X, Y):[0,1] \rightarrow \mathscr{L}\left(\mathbb{R}^{k}, \mathbb{R}^{2 k}\right)$, where

$$
X=\left(\begin{array}{ll}
\bar{X} & A \\
0 & \mathbb{1}
\end{array}\right), \quad Y=\left(\begin{array}{ll}
\bar{Y} & B \\
C & D
\end{array}\right)
$$

and thus a Lagrangian frame for $\bar{\Lambda}_{0}$ is given by $\bar{Z}=(\bar{X}, \bar{Y}):[0,1] \rightarrow \mathscr{L}\left(\mathbb{R}^{n}, \mathbb{R}^{2 n}\right)$. We compute the crossing form for the pair $\left(\Lambda_{0}, \Lambda_{1}\right)$.

A crossing for $\left(\Lambda_{0}, \Lambda_{1}\right)$ is a number $t \in[0,1]$ such that there is a $u=\left(u^{\prime}, 0\right) \in \mathbb{R}^{k}$ such that $\bar{Y}(t) u^{\prime}=0$, that is, if and only if $t$ is a crossing for $\left(\bar{\Lambda}_{0}, \bar{\Lambda}_{1}\right)$. At a crossing $t$ the crossing form is given by

$$
\begin{aligned}
\Gamma\left(\Lambda_{0}, \Lambda_{1}, t\right)(v) & =\langle X(t) u, \dot{Y}(t) u\rangle-\langle Y(t) u, \dot{X}(t) u\rangle \\
& =\left\langle\bar{X}(t) u^{\prime}, \dot{\bar{Y}}(t) u^{\prime}\right\rangle=\Gamma\left(\bar{\Lambda}_{0}, \bar{\Lambda}_{1}, t\right)\left(v^{\prime}\right),
\end{aligned}
$$

where $v=Z(t) u, v^{\prime}=\bar{Z}(t) u^{\prime}$. This proves the lemma.

PROOF OF LEMMA 7.1. In view of Lemma 7.2 it is sufficient to show that

$$
\mu\left(\Psi_{c, H} \Lambda_{0}, \Lambda_{1}\right)=\mu\left(\Psi_{c, H} \Lambda_{0}, \Lambda_{1}(1)\right)+\frac{1}{2} \operatorname{sign} I I_{c(1)} .
$$

Let $\Phi:[0,1] \rightarrow \operatorname{Sp}(2 k)$ be a path of symplectic matrices satisfying

$$
\Lambda_{1}(t)=\Phi(t) \Lambda_{1}(1)
$$

and abbreviate $\Psi=\Psi_{c, H}$. Then

$$
\begin{aligned}
\mu\left(\Psi \Lambda_{0}, \Lambda_{1}\right) & =\mu\left(\Psi \Lambda_{0}, \Phi \Lambda_{1}(1)\right) \\
& =\mu\left(\Phi^{-1} \Psi \Lambda_{0}, \Lambda_{1}(1)\right) \\
& =\mu\left(\Phi(1)^{-1} \Psi \Lambda_{0}, \Lambda_{1}(1)\right)+\mu\left(\Phi^{-1} \Psi(0) \Lambda_{0}, \Lambda_{1}(1)\right) \\
& =\mu\left(\Psi \Lambda_{0}, \Lambda_{1}(1)\right)+\mu\left(\Lambda_{0}, \Lambda_{1}\right) .
\end{aligned}
$$


By multiplying by a path of unitary matrices if necessary we may assume that

$$
\Lambda_{1}(t)=\left\{(\xi, 0,-B(t) \xi, \eta) \in \mathbb{R}^{2 k}: \xi \in \mathbb{R}^{n}, \eta \in \mathbb{R}^{k-n}\right\},
$$

where $B:[0,1] \rightarrow \mathbb{R}^{n \times n}$ is a path of symmetric matrices with $B(0)=0$. Now by the product and localisation properties of the Maslov index

$$
\mu\left(\Lambda_{0}, \Lambda_{1}\right)=\frac{1}{2} \operatorname{sign} B(1)=\frac{1}{2} \operatorname{sign} I_{c(1)} .
$$

This proves the lemma.

\section{The signature identity}

In this section we generalise the signature theorem of Robbin and Salamon in [14] to the case of nondegenerate critical points of the action functional on the 1 -jet bundle of a closed manifold.

THEOREM 8.1. Let $c \in \mathscr{P}$ be a nondegenerate critical point of $\Phi, \bar{c}$ its projection to $\mathscr{P}_{M}$ and $c^{N} \in \mathscr{P}^{N}$ the corresponding critical point of $\Phi^{N}$. Then, for sufficiently large $N$, the signature of the second variation of $\Phi^{N}$ at $c^{N}$ is given by

$$
\operatorname{sign} A^{N}\left(c^{N}\right)=2 \mu(\bar{c}, \bar{H}) \text {. }
$$

This strengthens the theorem of Viterbo [17], which states that, in the symplectic case, the difference of the signature of the second variation of $\Phi^{N}$ at two critical points is independent of $N$, but at the cost of having to take $N$ sufficiently large.

Below we prove a result which implies this theorem.

Let $G:[0,1] \times J^{1} \mathbb{R}^{k} \rightarrow \mathbb{R}$ be a quadratic Hamiltonian and denote by $\Psi_{G}:[0,1] \rightarrow$ $\mathrm{Sp}(2 k)$ the associated path of symplectic matrices given by (14). Also, let $F \subset \mathbb{R}^{k}$ be a linear subspace and $B: F \rightarrow F$ a linear transformation such that $\left\langle B \xi, \xi^{\prime}\right\rangle=\left\langle\xi, B \xi^{\prime}\right\rangle$ for all $\xi, \xi^{\prime} \in F$. Define the Lagrangian subspace

$$
\Lambda_{F, B}=\left\{(\xi, \eta) \in \mathbb{R}^{2 k}: \xi \in F, B \xi+\Pi_{F} \eta\right\},
$$

where $\Pi_{F} \in \mathbb{R}^{k \times k}$ denotes the orthogonal projection onto $F$. Now define the function $\Phi_{G, F, B}^{N}: W_{F}^{N} \rightarrow \mathbb{R}$ by

$$
\Phi_{G, F, B}^{N}(\gamma)=\sum_{j=1}^{N}\left(\left\langle\eta_{j}, \xi_{j}-\xi_{j-1}\right\rangle-W_{j-1}\left(\xi_{j-1}, \eta_{j}, \zeta_{j-1}\right)\right)+\frac{1}{2}\left\langle B \xi_{N}, \xi_{N}\right\rangle
$$

where

$$
W_{F}^{N}=\left\{\gamma=\left(\xi_{0}, \ldots, \xi_{N}, \eta_{1}, \ldots, \eta_{N}\right) \in \mathbb{R}^{(2 N+1) k} \mid \xi_{N} \in F\right\}
$$


and where the $W_{j} s$ are computed using (23), and the $\zeta_{j}$ s using (26). Denote by $A_{G, F, B}^{N}$ the second variation of $\Phi_{G, F, B}^{N}$.

In view of Proposition 6.10 and Lemma 7.1, Theorem 8.1 now follows from:

THEOREM 8.2. Let $G, F$ and $B$ be as above and assume that $\Psi_{G}(1) \Lambda_{0}$ is transverse to $\Lambda_{F, B}$. Then, for $N$ sufficiently large, the signature of the second variation of $\Phi_{G, F, B}^{N}$ is given by

$$
\operatorname{sign} A_{G, F, B}^{N}=2 \mu\left(\Psi_{G} \Lambda_{0}, \Lambda_{F, B}\right)+\operatorname{sign} B .
$$

The proof we give is based on that given by Robbin and Salamon [14] in the case where $F=\mathbb{R}^{k}, B=0$ and $G$ is independent of $z$.

Proof. Without loss of generality assume that

$$
\Lambda_{F, B}=\left\{\left(\xi^{\prime}, 0,-B \xi^{\prime}, \eta^{\prime \prime}\right) \in \mathbb{R}^{n} \times \mathbb{R}^{k-n} \times \mathbb{R}^{n} \times \mathbb{R}^{k-n}=\mathbb{R}^{2 k}: \xi^{\prime} \in \mathbb{R}^{n}, \eta^{\prime \prime} \in \mathbb{R}^{k-n}\right\}
$$

where $\operatorname{dim} F=n$ and where $B \in \mathbb{R}^{n \times n}$ is symmetric.

The proof of the theorem now proceeds in three steps.

STEP 1 . The theorem holds in the case

$$
G\left(t, \xi^{\prime}, \xi^{\prime \prime}, \eta^{\prime}, \eta^{\prime \prime}, \zeta\right)=\frac{1}{2}\left\langle G_{\xi^{\prime} \xi^{\prime}}(t) \xi^{\prime}, \xi^{\prime}\right\rangle .
$$

In this case the path of symplectic matrices associated to $G$ is given by

$$
\Psi_{G}(t)=\left(\begin{array}{cccc}
\mathbb{1} & 0 & 0 & 0 \\
0 & \mathbb{1} & 0 & 0 \\
C(t) & 0 & \mathbb{1} & 0 \\
0 & 0 & 0 & \mathbb{1}
\end{array}\right),
$$

where

$$
C(t)=\int_{0}^{t}-G_{\xi^{\prime} \xi^{\prime}}(\tau) d \tau .
$$

Thus, from the product and the localisation properties of the Maslov index,

$$
\mu\left(\Psi_{G} \Lambda_{0}, \Lambda_{F, B}\right)=\frac{1}{2} \operatorname{sign}(C(1)+B)-\frac{1}{2} \operatorname{sign} B .
$$

Also, from (23),

$$
W_{j}\left(\xi_{j}, \eta_{j+1}, \zeta_{j}\right)=\frac{1}{2}\left\langle W_{j, \xi^{\prime} \xi^{\prime}} \xi_{j}^{\prime}, \xi_{j}^{\prime}\right\rangle
$$

and defining

$$
C^{j}=\sum_{i=0}^{j-1}-W_{i, \xi^{\prime} \xi^{\prime}}
$$


for $j=1, \ldots, N$, it is easy to see that $C^{N}=C(1)$. Hence the second variation is given by

$$
\left\langle A_{G, F, B}^{N} \gamma, \gamma\right\rangle=2 \sum_{j=1}^{N}\left\langle\eta_{j}, \xi_{j}-\xi_{j-1}\right\rangle-\sum_{j=0}^{N-1}\left\langle W_{j, \xi^{\prime} \xi^{\prime}} \xi_{j}^{\prime}, \xi_{j}^{\prime}\right\rangle+\left\langle B \xi_{N}^{\prime}, \xi_{N}^{\prime}\right\rangle .
$$

Now define the following system of coordinates on $W_{F}^{N}=\mathbb{R}^{2 N k+n}$ :

$$
\begin{gathered}
u_{j}^{\prime}=\xi_{j}^{\prime}-\xi_{j-1}^{\prime}, \quad u_{j}^{\prime \prime}=\xi_{j}^{\prime \prime}-\xi_{j-1}^{\prime \prime}, \\
v_{j}^{\prime}=\eta_{j}^{\prime}-\frac{1}{2} C^{j}\left(\xi_{j}^{\prime}+\xi_{j-1}^{\prime}\right), \quad v_{j}^{\prime \prime}=\eta_{j}^{\prime \prime}, \quad w=\xi_{N}^{\prime}
\end{gathered}
$$

and denote $u=\left(u_{1}^{\prime}, u_{1}^{\prime \prime}, \ldots, u_{N}^{\prime}, u_{N}^{\prime \prime}\right), v=\left(v_{1}^{\prime}, v_{1}^{\prime \prime}, \ldots, v_{N}^{\prime}, v_{N}^{\prime \prime}\right)$. In these coordinates the $L^{2}$-inner product of $u$ and $v$ is given by

$$
\begin{aligned}
\langle u, v\rangle & =\sum_{j=1}^{N}\left\langle u_{j}^{\prime}, v_{j}^{\prime}\right\rangle+\sum_{j=1}^{N}\left\langle u_{j}^{\prime \prime}, v_{j}^{\prime \prime}\right\rangle \\
& =\sum_{j=1}^{N}\left\langle\xi_{j}^{\prime}-\xi_{j-1}^{\prime}, \eta_{j}^{\prime}\right\rangle-\frac{1}{2} \sum_{j=1}^{N}\left\langle\xi_{j}^{\prime}-\xi_{j-1}^{\prime}, C^{j}\left(\xi_{j}^{\prime}+\xi_{j-1}^{\prime}\right)\right\rangle+\sum_{j=1}^{N}\left\langle\xi_{j}^{\prime \prime}-\xi_{j-1}^{\prime \prime}, \eta_{j}^{\prime \prime}\right\rangle \\
& =\sum_{j=1}^{N}\left\langle\xi_{j}-\xi_{j-1}, \eta_{j}\right\rangle+\frac{1}{2} \sum_{j=1}^{N}\left\langle C^{j} \xi_{j-1}^{\prime}, \xi_{j-1}^{\prime}\right\rangle-\frac{1}{2} \sum_{j=1}^{N}\left\langle C^{j} \xi_{j}^{\prime}, \xi_{j}^{\prime}\right\rangle \\
& =\sum_{j=1}^{N}\left\langle\xi_{j}-\xi_{j-1}, \eta_{j}\right\rangle+\frac{1}{2} \sum_{j=0}^{N-1}\left\langle C^{j+1} \xi_{j}^{\prime}, \xi_{j}^{\prime}\right\rangle-\frac{1}{2} \sum_{j=0}^{N}\left\langle C^{j} \xi_{j}^{\prime}, \xi_{j}^{\prime}\right\rangle \\
& =\sum_{j=1}^{N}\left\langle\xi_{j}-\xi_{j-1}, \eta_{j}\right\rangle+\frac{1}{2} \sum_{j=0}^{N-1}\left\langle\left(C^{j+1}-C^{j}\right) \xi_{j}^{\prime}, \xi_{j}^{\prime}\right\rangle-\frac{1}{2}\left\langle C^{N} \xi_{N}^{\prime}, \xi_{N}^{\prime}\right\rangle \\
& =\sum_{j=1}^{N}\left\langle\xi_{j}-\xi_{j-1}, \eta_{j}\right\rangle-\frac{1}{2} \sum_{j=0}^{N-1}\left\langle W_{j, \xi^{\prime} \xi^{\prime}} \xi_{j}^{\prime}, \xi_{j}^{\prime}\right\rangle-\frac{1}{2}\left\langle C^{N} \xi_{N}^{\prime}, \xi_{N}^{\prime}\right\rangle \\
& =\frac{1}{2}\left\langle A_{G, F}^{N} \gamma, \gamma\right\rangle-\frac{1}{2}\left\langle\left(C^{N}+B\right) w, w\right\rangle .
\end{aligned}
$$

Thus the second variation satisfies

$$
\frac{1}{2}\left\langle A_{G, F, B}^{N} \gamma, \gamma\right\rangle=\langle u, v\rangle+\frac{1}{2}\left\langle\left(C^{N}+B\right) w, w\right\rangle
$$

and hence

$$
\operatorname{sign} A_{G, F, B}^{N}=\operatorname{sign}\left(C^{N}+B\right)=\operatorname{sign}(C(1)+B)=2 \mu\left(\Psi_{G} \Lambda_{0}, \Lambda_{F, B}\right)+\operatorname{sign} B .
$$

STEP 2. The theorem holds for quadratic Hamiltonians independent of $\zeta$. 
Let $G$ be a $\zeta$-independent Hamiltonian with $\Psi_{G}(1) \Lambda_{0}$ transverse to $\Lambda_{F, B}$ and suppose, for a given value of $k^{\prime}$, that $G^{\prime}: \mathbb{R}^{2 k^{\prime}} \rightarrow \mathbb{R}$ is any symplectic shear of the form

$$
G^{\prime}\left(\xi^{\prime}, \eta^{\prime}\right)=\frac{1}{2}\left\langle G_{\xi^{\prime} \xi^{\prime}}^{\prime} \xi^{\prime}, \xi^{\prime}\right\rangle
$$

where $G_{\xi^{\prime} \xi^{\prime}}^{\prime}$ is a nondegenerate symmetric matrix of signature zero. (It follows that $k^{\prime}$ is necessarily even.) From Step 1 it follows that

$$
\operatorname{sign} A_{G^{\prime}, \mathbb{R}^{k^{\prime}, 0}}^{N}=2 \mu\left(\Psi_{G^{\prime}} \Lambda_{0}^{\prime}, \Lambda_{0}^{\prime}\right)=0,
$$

where $\Lambda_{0}^{\prime}=\mathbb{R}^{k^{\prime}} \times\{0\}$. Now define $G_{0}=G \oplus G^{\prime}:[0,1] \times J^{1} \mathbb{R}^{k+k^{\prime}} \rightarrow \mathbb{R}$ by

$$
G_{0}\left(t, \xi, \xi^{\prime}, \eta, \eta^{\prime}, \zeta\right)=G(t, \xi, \eta, \zeta)+G^{\prime}\left(\xi^{\prime}, \eta^{\prime}\right)
$$

By additivity of the signature

$$
\operatorname{sign} A_{G_{0}, F \oplus \mathbb{R}^{k^{\prime}, B \oplus 0}}^{N}=\operatorname{sign} A_{G . F . B}^{N}
$$

and by the product property of the Maslov index

$$
\mu\left(\Psi_{G_{0}} \Lambda_{0} \oplus \Lambda_{0}^{\prime}, \Lambda_{F, B} \oplus \Lambda_{0}^{\prime}\right)=\mu\left(\Psi_{G} \Lambda_{0}, \Lambda_{F, B}\right) .
$$

Hence it suffices to prove the theorem for $G_{0}$ with $F$ replaced by $\widetilde{F}=F \oplus \mathbb{R}^{k^{\prime}}$ and $B$ by $\widetilde{B}=B \oplus 0$.

Now, for $k^{\prime}$ sufficiently large, let $G_{1}$ be a symplectic shear of the form considered in Step 1, with $F$ replaced by $\widetilde{F}$, satisfying

$$
\mu\left(\Psi_{G_{1}} \widetilde{\Lambda}_{0}, \Lambda_{\tilde{F}, \tilde{B}}\right)=\mu\left(\Psi_{G_{0}} \widetilde{\Lambda}_{0}, \Lambda_{\widetilde{F}, \widetilde{B}}\right),
$$

where $\tilde{\Lambda}_{0}=\Lambda_{0} \oplus \Lambda_{0}^{\prime}$. This exists by the localisation and integrality properties of the Maslov index. Also, choose a symplectic matrix $\Phi \in \mathrm{Sp}\left(2 k+2 k^{\prime}\right)$ such that $\Lambda_{\tilde{F}, \tilde{B}}=\Phi \tilde{\Lambda}_{0}$. Then

$$
\mu\left(\Phi^{-1} \Psi_{G_{1}} \widetilde{\Lambda}_{0}, \widetilde{\Lambda}_{0}\right)=\mu\left(\Phi^{-1} \Psi_{G_{0}} \widetilde{\Lambda}_{0}, \widetilde{\Lambda}_{0}\right)
$$

and the symplectic path $\Phi^{-1} \Psi_{G_{i}}$ satisfies

$$
\Phi^{-1} \Psi_{G_{i}}(0)=\Phi^{-1}, \quad \Phi^{-1} \Psi_{G_{i}}(1) \tilde{\Lambda}_{0} \cap \tilde{\Lambda}_{0}=\{0\} .
$$

Thus by the homotopy property of the Maslov index there exists a homotopy of symplectic paths $\Phi^{-1} \Psi_{\lambda}:[0,1] \rightarrow \operatorname{Sp}\left(2 k+2 k^{\prime}\right)$ between $\Phi^{-1} \Psi_{G_{0}}$ and $\Phi^{-1} \Psi_{G_{1}}$ and within the same class. From this we can construct a quadratic Hamiltonian $G_{\lambda}$ such that $\Psi_{\lambda}=\Psi_{G_{\lambda}}$. 
Now choose $N$ sufficiently large such that for each $\lambda$ the function $\Phi_{G_{\lambda}, \tilde{F}, \tilde{B}}^{N}$ is defined. Then

$$
\begin{aligned}
\operatorname{sign} A_{G_{0}, \widetilde{F}, \widetilde{B}}^{N}=\operatorname{sign} A_{G_{1}, \widetilde{F}, \widetilde{B}}^{N} & =2 \mu\left(\Psi_{G_{1}} \widetilde{\Lambda}_{0}, \Lambda_{\widetilde{F}, \widetilde{B}}\right)+\operatorname{sign} \widetilde{B} \\
& =2 \mu\left(\Psi_{G_{0}} \widetilde{\Lambda}_{0}, \Lambda_{\widetilde{F}, \widetilde{B}}\right)+\operatorname{sign} \widetilde{B} .
\end{aligned}
$$

Here the first equality follows from Corollary 6.12.

STEP 3. The general case.

To the quadratic Hamiltonian

$$
G(t, \xi, \eta, \zeta)=\frac{1}{2}\left\langle G_{\xi \xi}(t) \xi, \xi\right\rangle+\left\langle G_{\eta \xi}(t) \xi, \eta\right\rangle+\frac{1}{2}\left\langle G_{\eta \eta}(t) \eta, \eta\right\rangle+G_{\zeta}(t) \zeta
$$

associate the quadratic $\zeta$-independent Hamiltonian

$$
G^{\prime}(t, \xi, \eta, \zeta)=\frac{1}{2}\left\langle G_{\xi \xi}(t) \xi, \xi\right\rangle+\left\langle\left(G_{\eta \xi}(t)+\frac{1}{2} G_{\zeta}(t) \mathbb{1}\right) \xi, \eta\right\rangle+\frac{1}{2}\left\langle G_{\eta \eta}(t) \eta, \eta\right\rangle .
$$

Now consider the homotopy $G_{\lambda}=\lambda G^{\prime}+(1-\lambda) G$ between $G$ and $G^{\prime}$. Notice that $\Psi_{G_{\lambda}}=\Psi_{G}$ as symplectic paths for all $\lambda$. Step 3 now follows from Corollary 6.12 . This completes the proof of the theorem.

\section{Proof of the generalised Morse inequalities}

Recall from Remark 6.5 that the discrete action functional $\Phi^{N}: M \times \mathbb{R}^{2 m} \rightarrow \mathbb{R}$ can be written in the form

$$
\Phi^{N}(x, \xi)=\frac{1}{2}\langle P \xi, \xi\rangle+W(x, \xi),
$$

where $P \in \mathbb{R}^{2 m \times 2 m}$ is a nondegenerate symmetric matrix of signature zero and where the gradient of $W$ in the direction of the fibre, $\partial_{\xi} W$, is bounded. We prove the generalised Morse inequalities by studying the critical points of $\Phi^{N}$. As

$M \times \mathbb{R}^{2 m}$ is noncompact stabilised Morse theory will be used; see Conley [6].

In order to study the critical points of $\Phi^{N}$ we consider the (negative) gradient flow. This is defined by the equations

$$
\frac{d \xi}{d s}=-P \xi-\frac{\partial W}{\partial \xi}(x, \xi), \quad \frac{d x}{d s}=-\frac{\partial W}{\partial x}(x, \xi) .
$$

Now induced by $P$ there is a splitting

$$
\mathbb{R}^{2 m}=E^{-} \oplus E^{+}
$$

into the negative and positive eigenspaces. It follows that, for some $\delta>0$,

$$
\left\langle P \xi^{-}, \xi^{-}\right\rangle \leq-\delta\left|\xi^{-}\right|^{2}, \quad\left\langle P \xi^{+}, \xi^{+}\right\rangle \geq \delta\left|\xi^{+}\right|^{2}
$$


for $\xi^{-} \in E^{-}, \xi^{+} \in E^{+}$. Thus we can find constants $\varepsilon>0$ and $R>0$ such that

$$
\begin{array}{lll}
\frac{d}{d s}\left|\xi^{-}\right|^{2} \geq \varepsilon & \text { if } & \left|\xi^{-}\right| \geq R, \\
\frac{d}{d s}\left|\xi^{+}\right|^{2} \leq-\varepsilon & \text { if } & \left|\xi^{+}\right| \geq R .
\end{array}
$$

Indeed, for $\left|\xi^{-}\right| \geq R$ and $R$ sufficiently large,

$$
\frac{d}{d s} \frac{\left|\xi^{-}\right|^{2}}{2}=\left\langle\xi^{-},-P \xi^{-}-\partial_{\xi} W\right\rangle \geq \delta\left|\xi^{-}\right|^{2}-\sup \left|\partial_{\xi} W\right| \cdot\left|\xi^{-}\right| \geq \varepsilon,
$$

and similarly for $\left|\xi^{+}\right|^{2} \geq R$. Thus an isolating block, in the sense of Conley, for the compact invariant set $\Lambda$ of all bounded orbits of the gradient flow, is given by

$$
N=\left\{\left(x, \xi^{-}+\xi^{+}\right): \xi^{ \pm} \in E^{ \pm},\left|\xi^{ \pm}\right| \leq R\right\}
$$

with exit set

$$
L=\left\{\left(x, \xi^{-}+\xi^{+}\right) \in N:\left|\xi^{-}\right|=R\right\}
$$

for $R$ sufficiently large.

We use the following notation

$$
\begin{aligned}
b_{k}(\Lambda) & =\operatorname{dim} H_{k}(N, L), \\
b_{k}(M) & =\operatorname{dim} H_{k}(M), \\
c_{k}(\Lambda) & =\#\left\{c \in \Lambda \mid d \Phi^{N}(c)=0, \operatorname{ind}_{\Phi^{N}}(c)=k\right\}, \\
p_{k}(\Lambda) & =\#\left\{c \in \Lambda \mid d \Phi^{N}(c)=0, \mu(c, \bar{H})=k\right\},
\end{aligned}
$$

where ind $_{\Phi^{N}}(c)$ denotes the Morse index of $\Phi^{N}$ at $c$ and is defined to be the number of negative eigenvalues of the Hessian $d^{2} \Phi^{N}(c)$. Here the numbers $b_{k}(\Lambda)$ are known as the Conley-Betti numbers. These are related to the $c_{k}(\Lambda)$ by the Morse inequalities.

THEOREM 9.1 (Morse inequalities). For $k=0, \ldots, n+2 m$

$$
c_{k}(\Lambda)-c_{k-1}(\Lambda)+\cdots \pm c_{0}(\Lambda) \geq b_{k}(\Lambda)-b_{k-1}(\Lambda)+\cdots \pm b_{0}(\Lambda)
$$

with equality holding for $k=n+2 m$.

These inequalities are proved in [12].

The Conley-Betti numbers are related to the Betti numbers of $M$ by the Thom isomorphism. Specifically, denote by $E^{m}$ the $m$-dimensional Euclidean space, by $B^{m}$ and $S^{m-1}$ the closed unit ball and unit sphere respectively in $E^{m}$, and abbreviate $E_{0}^{m}=E^{m} \backslash\{0\}$. Then by homotopy

$$
\left(M \times B^{m} \times B^{m}, M \times \partial B^{m} \times B^{m}\right) \simeq\left(M \times B^{m}, M \times S^{m-1}\right) \simeq\left(M \times E^{m}, M \times E_{0}^{m}\right) .
$$


Thus

$$
H_{k}(N, L)=H_{k}\left(M \times E^{m}, M \times E_{0}^{m}\right)=H_{k-m}(M)
$$

where the last equality is the Thom isomorphism. In other words,

$$
b_{k}(\Lambda)=b_{k-m}(M)
$$

Also by Theorem 8.1

$$
c_{k}=p_{n / 2+m-k}
$$

The proof of Theorem 1.1, the generalised Morse inequalities, is now completed by putting together (29), (30) and the Morse inequalities.

\section{Acknowledgements}

The author would like to thank his doctoral supervisor, D. Salamon, for his help. This paper was prepared at the Max-Planck-Institut für Mathematik, Bonn.

\section{References}

[1] V. I. Arnold, 'First steps in symplectic topology', Russian Math. Surveys 41 (1986), 1-21.

[2] M. Brunella, 'On a theorem of Sikorav', Enseign. Math. (2) 37 (1991), 83-87.

[3] M. Chaperon, 'Une idée du type 'géodésiques brisées' pour les systemes Hamiltoniens', $C . R$. Acad. Sci. Paris Sér. I Math. 298 (1984), 293-296.

[4] — . 'On generating families', in: The Floer Memorial Volume (eds. H. Hofer, C. Taubes, A. Weinstein and E. Zehnder) (Birkäuser, Basel, 1996) pp. 283-296.

[5] Yu. V. Chekanov, 'Critical points of quasi-functions and generating families of Legendrian manifolds', Functional Anal. Appl. 30 (1996), 118-128.

[6] C. C. Conley, Isolated invariant sets and the Morse index, CBMS, Reg. Conf. Ser. in Math. 38 (American Math. Soc., Providence, RI, 1978).

[7] C. C. Conley and E. Zehnder, 'The Birkhoff-Lewis fixed point theorem and a conjecture of V. I. Arnold', Invent. Math. 73 (1983), 33-49.

[8] J. J. Duistermaat, 'On the Morse index theorem in variational calculus', Adv. Math. 21 (1976), 173-195.

[9] H. Hofer, 'Lagrangian embeddings and critical point theory', Ann. Inst. H. Poincaré Anal. NonLinéaire 2 (1985), 407-462.

[10] L. Hörmander, 'Fourier integral operators I', Acta Math. 127 (1971), 79-183.

[11] F. Laudenbach and J. C. Sikorav, 'Persistance d'intersection avec la section nulle au cours d'une isotopie Hamiltonienne dans un fibré cotangent', Invent. Math. 82 (1985), 349-357.

[12] D. McDuff and D. Salamon, Introduction to symplectic topology (Oxford University Press, Oxford, 1995).

[13] J. Robbin and D. Salamon, 'The Maslov index for paths', Topology 32 (1993), 827-844. 
[14] - 'Feynman path integrals on the phase space and the metaplectic representation', Math. $Z$. 221 (1996), 307-335.

[15] J. C. Sikorav, 'Problèmes d'intersection et de points fixes en géométrie Hamiltonienne', Comm. Math. Helvet. 62 (1987), 62-73.

[16] D. Théret, Utilisation des fonctions génératrices en géométrie symplectique globale (Ph.D. Thesis, Université Denis Diderot, Paris 7, 1995).

[17] C. Viterbo, 'Intersection de sous-variétés Lagrangiennes, fonctionnelles d'action et indice des systèmes Hamiltoniens', Bull. Soc. Math. France 115 (1987), 361-390.

[18] - 'Symplectic topology as the geometry of generating functions', Math. Ann. 292 (1992), 685-710.

\section{Mathematics Department}

Middle East Technical University

06531 Ankara

Turkey

e-mail: bhupal@math.metu.edu.tr 
